\title{
Quantum Mechanics-Based Structure Analysis of Cyclic Monoterpene Glycosides from Rhodiola rosea
}

Yu Tang, ${ }^{\dagger}$ J. Brent Friesen, ${ }^{\ddagger}$, David C. Lankin,$^{\dagger}$ James B. McAlpine, ${ }^{\dagger}$ Dejan Nikolić, ${ }^{\dagger}$ Matthias Niemitz, ${ }^{\perp}$ David S. Seigler, $\Perp$ James Graham, Shao-Nong Chen, ${ }^{\dagger}$, Guido F. Pauli ${ }^{\dagger, \ldots}, *$

'UIC/NIH Center for Botanical Dietary Supplements Research, Program for Collaborative Research in the Pharmaceutical Sciences (PCRPS) and Department of Pharmaceutical Sciences, College of Pharmacy, University of Illinois at Chicago, 833 S. Wood St., Chicago, IL 60612, United States

${ }^{\star}$ Center for Natural Product Technologies (CENAPT), Program for Collaborative Research in the Pharmaceutical Sciences (PCRPS) and Department of Pharmaceutical Sciences, College of Pharmacy, University of Illinois at Chicago, 833 S. Wood St., Chicago, IL 60612, United States

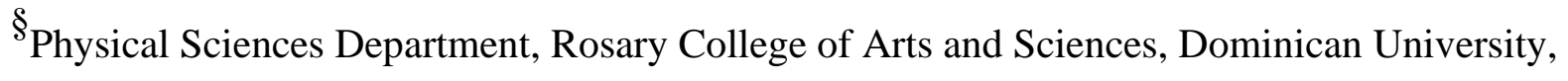
River Forest, IL 60305, United States

${ }^{\perp}$ NMR Solutions Ltd., Puijonkatu 24 B 5, 70110 Kuopio, Finland

$\|$ Department of Plant Biology, University of Illinois at Urbana Champaign, Urbana Champaign, IL 61801, United States

\section{Supporting Information}




\section{Table of Content}

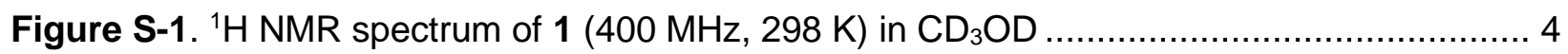

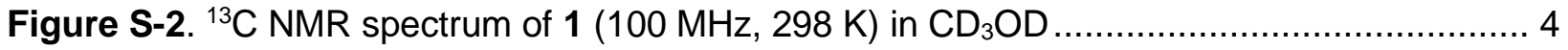

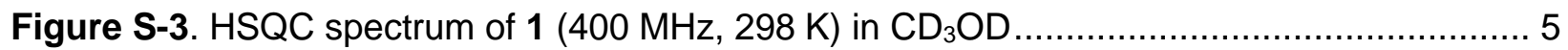

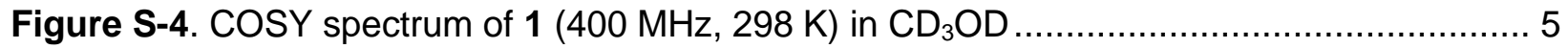

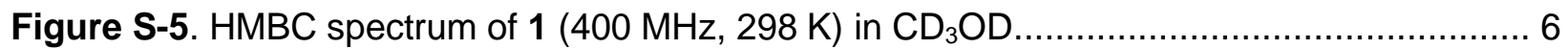

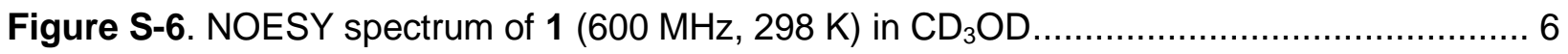

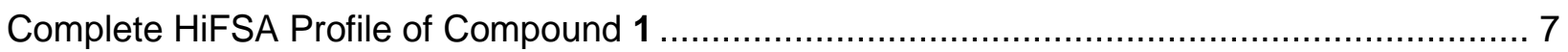

Figure S-7. The ${ }^{1} \mathrm{H}$ NMR fingerprint of 1 in $\mathrm{CD}_{3} \mathrm{OD}(400 \mathrm{MHz}, 298 \mathrm{~K})$ generated by PERCH .... 9

Figure S-8. The ${ }^{1} \mathrm{H}$ NMR fingerprint of 1 in $\mathrm{CD}_{3} \mathrm{OD}(400 \mathrm{MHz}, 298 \mathrm{~K})$ generated by CT.......... 9

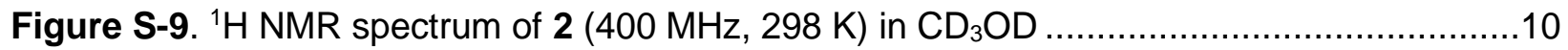

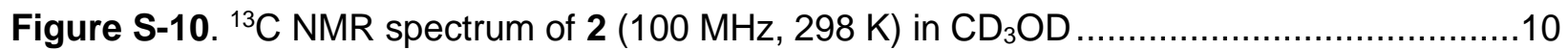

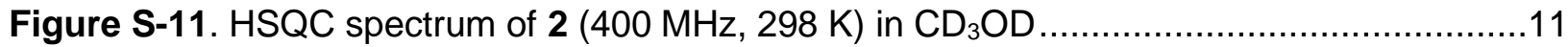

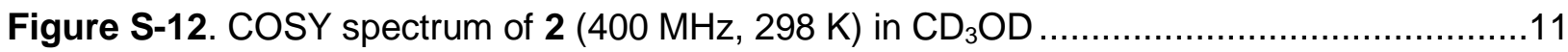

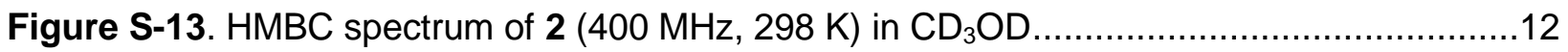

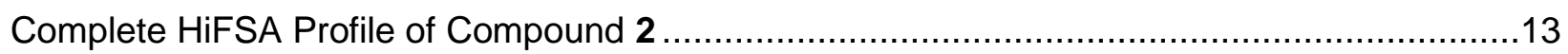

Figure S-14. The ${ }^{1} \mathrm{H}$ NMR fingerprint of 2 in $\mathrm{CD}_{3} \mathrm{OD}(400 \mathrm{MHz}, 298 \mathrm{~K})$ generated by $\mathrm{PERCH} .15$

Figure S-15. ${ }^{1} \mathrm{H}$ NMR spectrum of $3(400 \mathrm{MHz}, 298 \mathrm{~K})$ in $\mathrm{CD}_{3} \mathrm{OD}$.....................................16

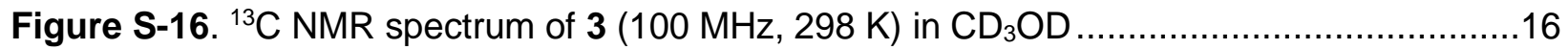

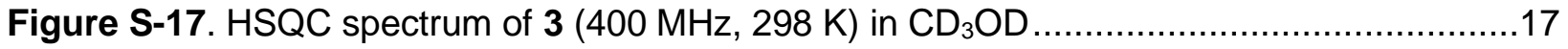

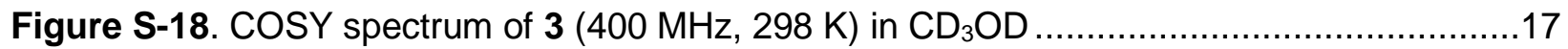

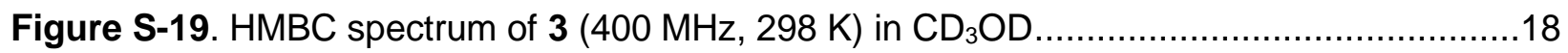

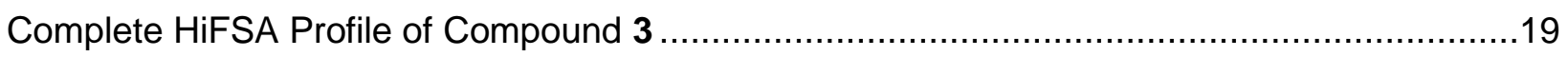

Figure S-20. The ${ }^{1} \mathrm{H}$ NMR fingerprint of 3 in $\mathrm{CD}_{3} \mathrm{OD}(400 \mathrm{MHz}, 298 \mathrm{~K})$ generated by $\mathrm{PERCH} .21$

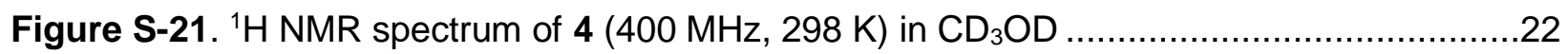

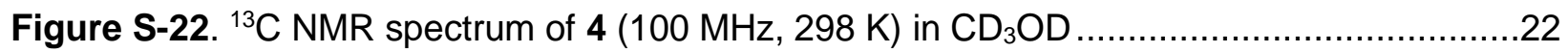

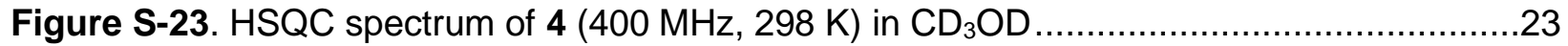

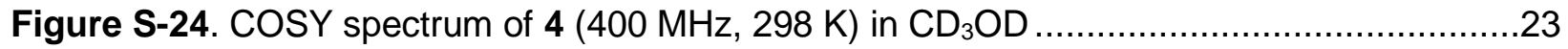

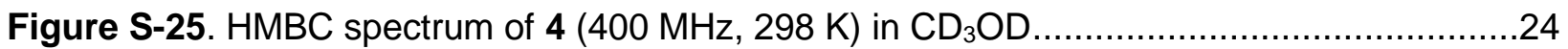

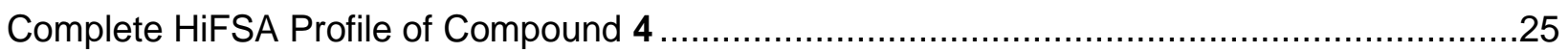

Figure S-26. The ${ }^{1} \mathrm{H}$ NMR fingerprint of 4 in $\mathrm{CD}_{3} \mathrm{OD}(400 \mathrm{MHz}, 298 \mathrm{~K})$ generated by $\mathrm{PERCH} .27$

Figure S-27. ${ }^{1} \mathrm{H}$ NMR spectra comparison (400 MHz, $298 \mathrm{~K}$ ) between hydrolyzed sugars and

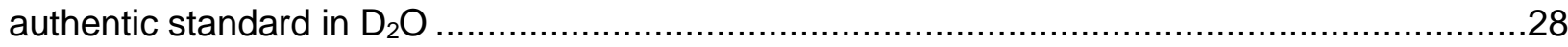




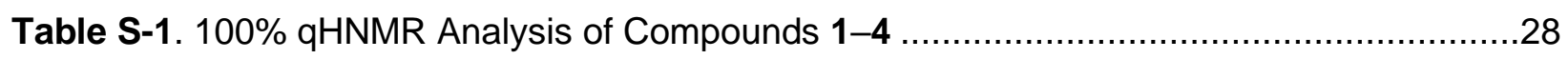

Table S-2. 100\% qHNMR Analysis of Detectable Impurities in Compounds 1-4..................29

Table S-3. $100 \%$ qHNMR Analysis of Hydrolyzed Sugar Mixture in Compounds 1 and 3 ........29 


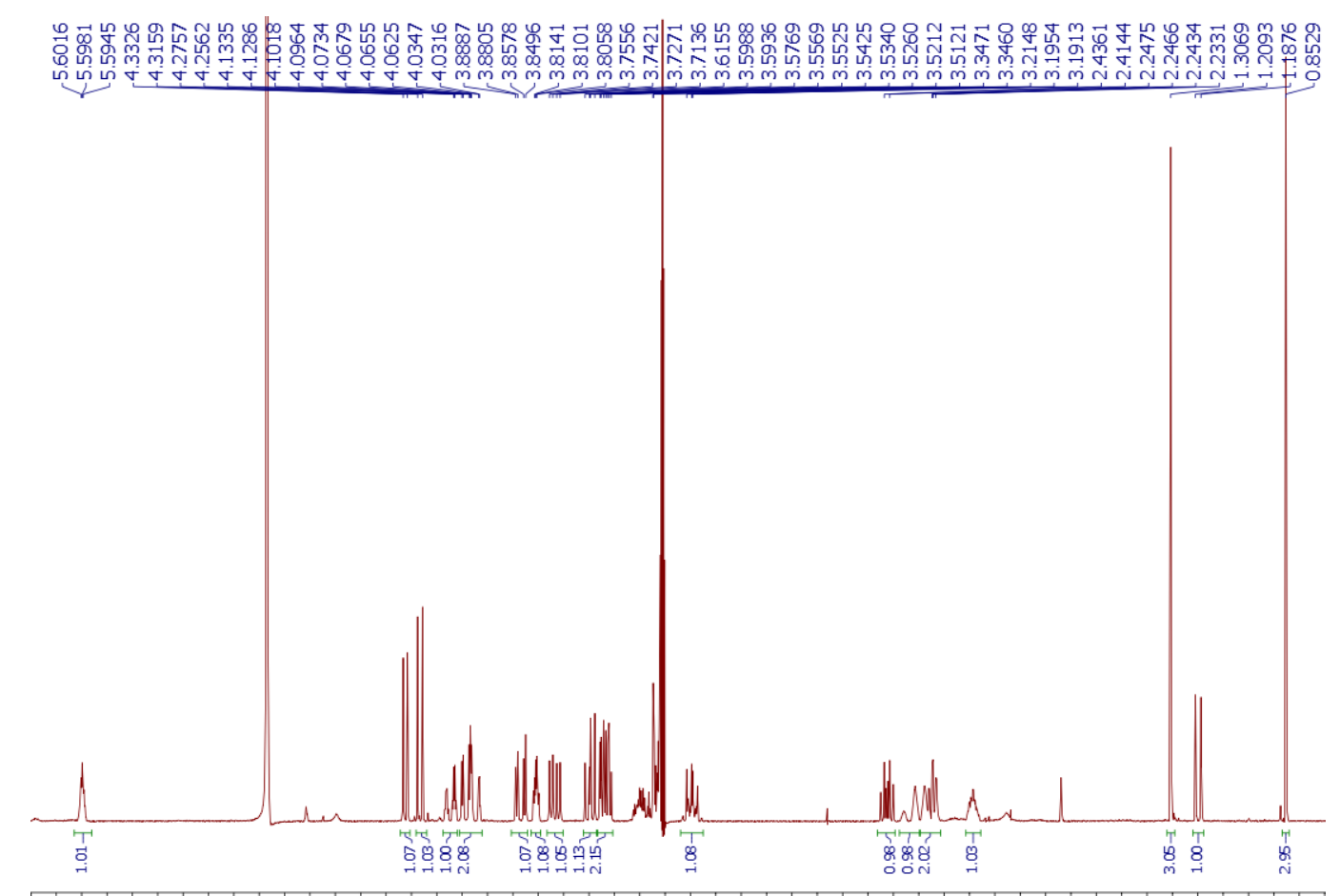

\begin{tabular}{lllllllllllllllllllllllllllllll}
\hline .8 & 5.6 & 5.4 & 5.2 & 5.0 & 4.8 & 4.6 & 4.4 & 4.2 & 4.0 & 3.8 & 3.6 & 3.4 & 3.2 & 3.0 & 2.8 & 2.6 & 2.4 & 2.2 & 2.0 & 1.8 & 1.6 & 1.4 & 1.2 & 1.0 & 0.8
\end{tabular}

Figure S-1. ${ }^{1} \mathrm{H}$ NMR spectrum of 1 (400 MHz, $298 \mathrm{~K}$ ) in $\mathrm{CD}_{3} \mathrm{OD}$

\begin{tabular}{|c|c|c|c|c|}
\hline శ & $\begin{array}{c}\frac{\pi}{5} \\
\frac{3}{1}\end{array}$ & 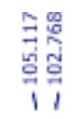 & 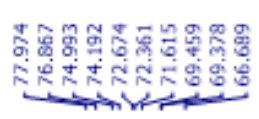 & 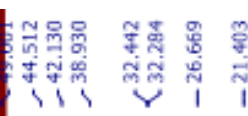 \\
\hline
\end{tabular}

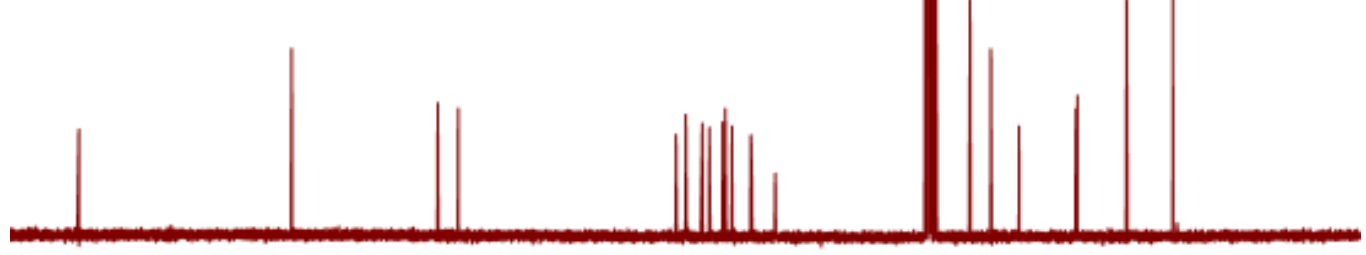

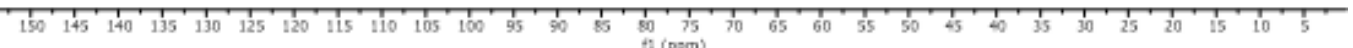

Figure S-2. ${ }^{13} \mathrm{C}$ NMR spectrum of $1(100 \mathrm{MHz}, 298 \mathrm{~K})$ in $\mathrm{CD}_{3} \mathrm{OD}$ 


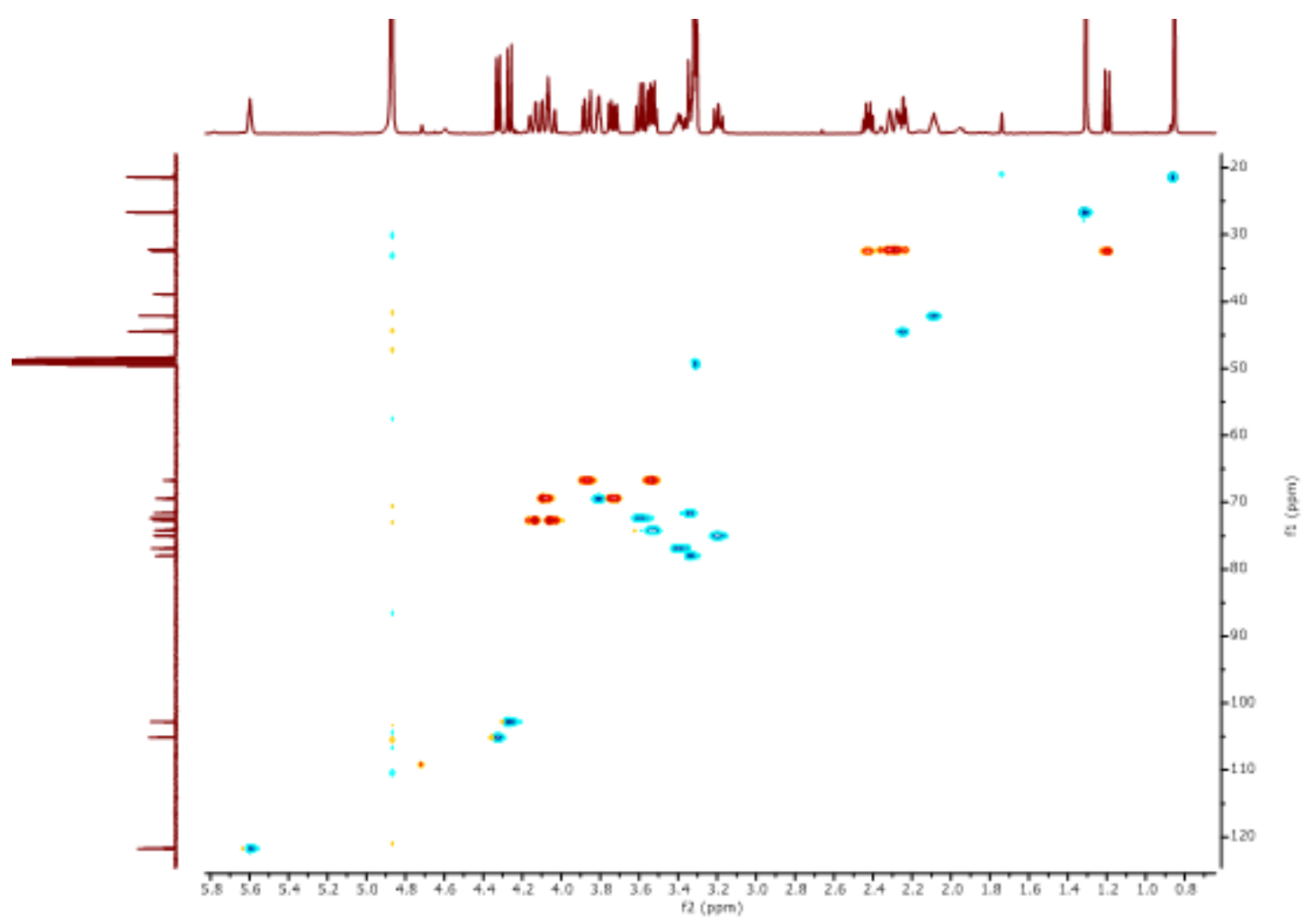

Figure S-3. HSQC spectrum of 1 (400 MHz, $298 \mathrm{~K}$ ) in $\mathrm{CD}_{3} \mathrm{OD}$

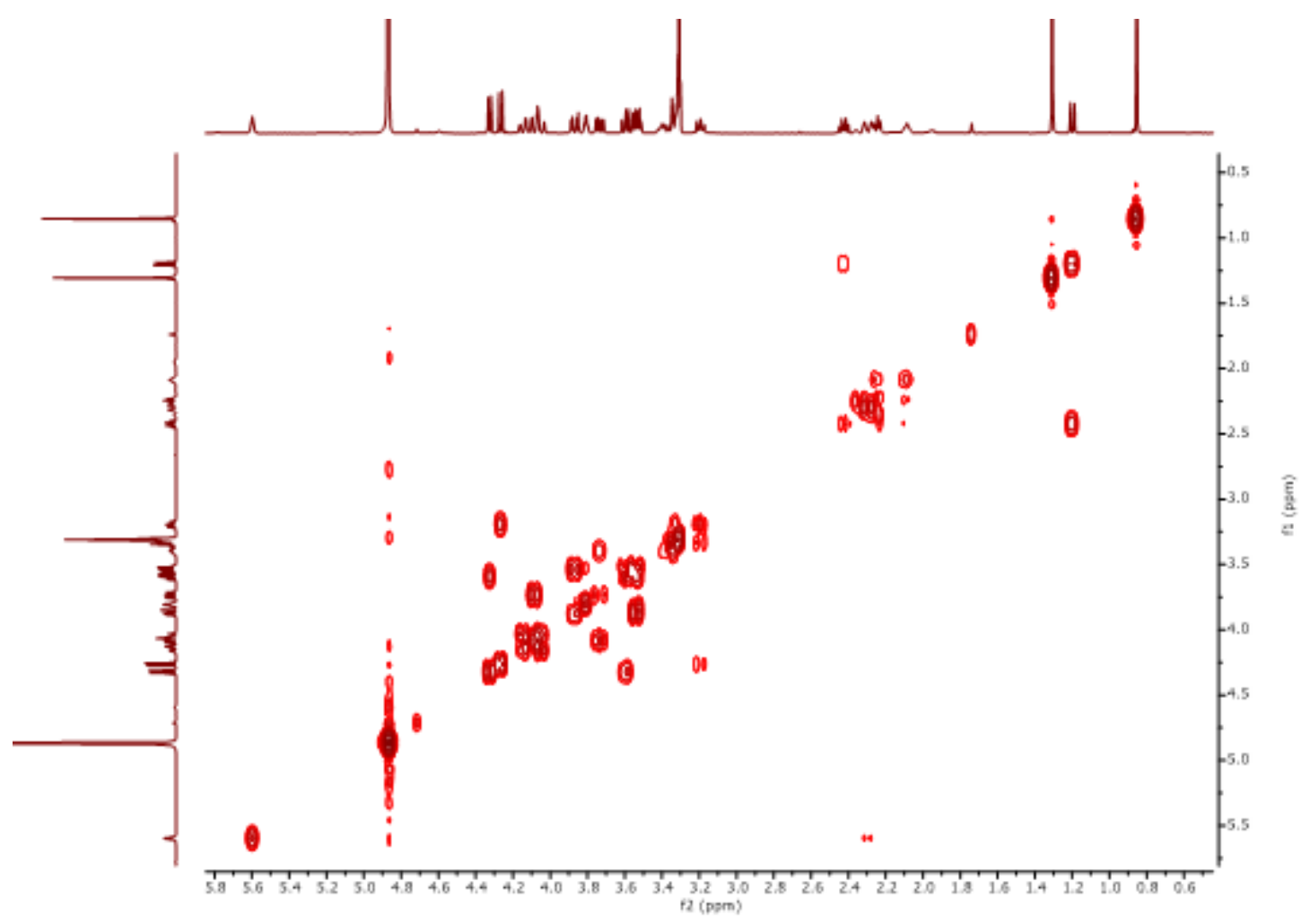

Figure S-4. COSY spectrum of 1 (400 MHz, $298 \mathrm{~K}$ ) in $\mathrm{CD}_{3} \mathrm{OD}$ 


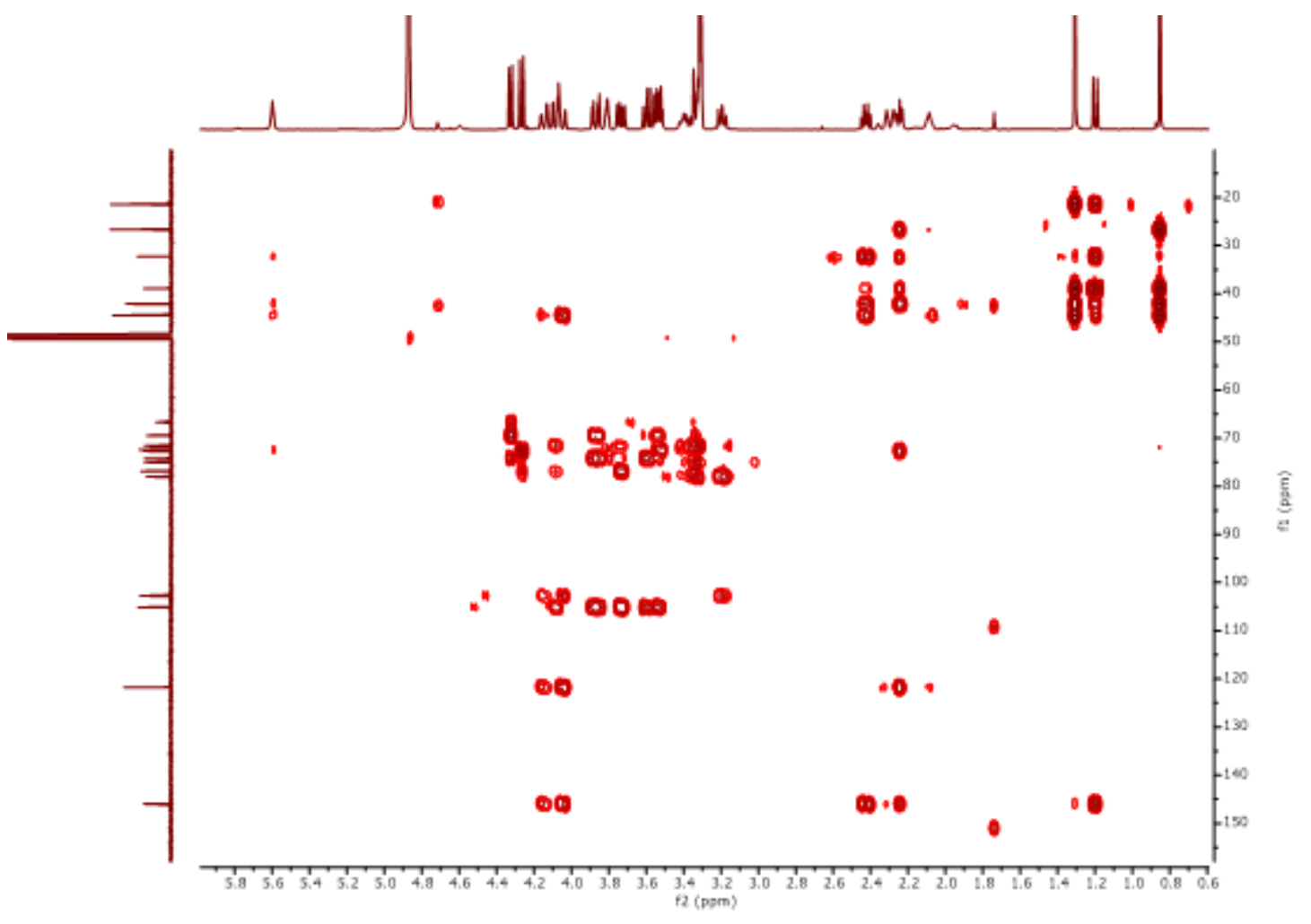

Figure S-5. $\mathrm{HMBC}$ spectrum of $1(400 \mathrm{MHz}, 298 \mathrm{~K})$ in $\mathrm{CD}_{3} \mathrm{OD}$

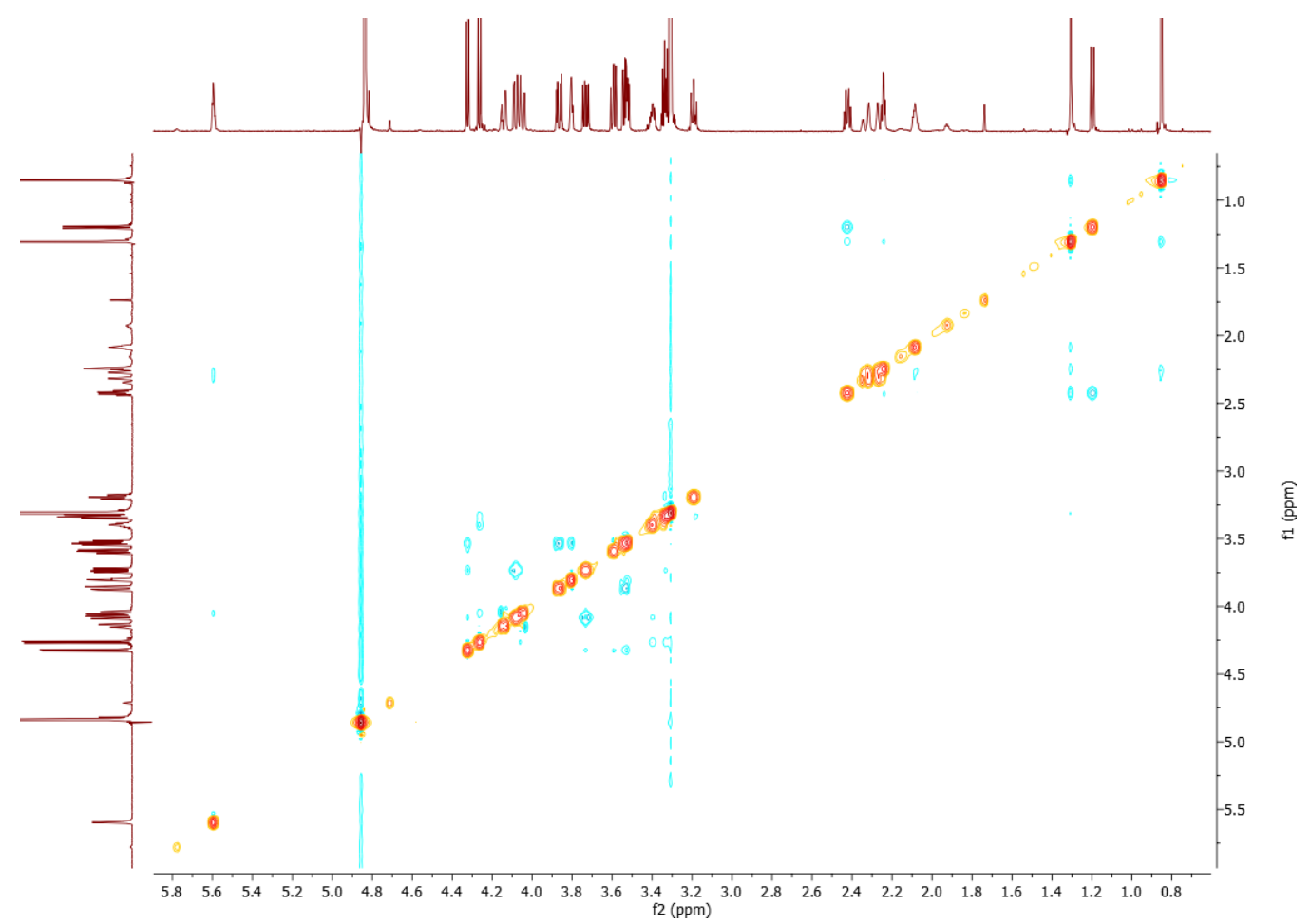

Figure S-6. NOESY spectrum of 1 (600 MHz, $298 \mathrm{~K}$ ) in $\mathrm{CD}_{3} \mathrm{OD}$ 


\section{Complete HiFSA Profile of Compound 1}

\footnotetext{
* NEW: the lines beginning by * are comment lines !

* To keep all the chemical shifts fixed during iteration

* replace "CHEMICAL SHIFTS(HZ):" by "..SHIFTS(HZ): fixed"

* The couplings can be fixed in the same way
}

ACTIVE SPECIES:1H

CHEMICAL SHIFTS(PPM):

PROTON 2*SPIN $=1$ SPECIES $=1 \mathrm{H} \quad$ POPULATION $(\mathrm{Y})=0.07225$

$\mathrm{H}-10 \mathrm{a} / \mathrm{1}$ 4.143956 $1 * 1 * 1$ STAT=Y PRED= 4.146 RANGE= 0.388 WIDTH(Y)=1.311 RESP(Y)=1.0000 HSQC= C-10 $\mathrm{H}-10 \mathrm{~b} / 1$ 4.051131 $1 * 1 * 1$ STAT $=\mathrm{Y}$ PRED= 4.048 RANGE= 0.388 WIDTH(Y)=1.245 RESP(Y)=1.0000 HSQC=C-10 H-1' / 1 4.265928 $1^{*}{ }^{\prime}{ }^{*} 1$ STAT $=Y$ PRED= 4.265 RANGE $=0.318$ WIDTH(Y)=0.866 RESP(Y)=1.0000 HSQC= C-1' H-5' / 13.400566 1*1*1 STAT=Y PRED= 3.403 RANGE $=0.213$ WIDTH(Y)=1.504 RESP(Y)=1.0000 HSQC= C-5' H-4' / $13.34017011^{*}{ }^{*} 1$ STAT=Y PRED= 3.339 RANGE= 0.238 WIDTH(Y)=1.081 RESP(Y)=1.0000 HSQC=C-4' H-3' / 13.330877 1*1*1 STAT=Y PRED= 3.333 RANGE=0.188 WIDTH(Y)=1.006 RESP(Y)=1.0000 HSQC= C-3' H-2' / $13.1945501 * 1 * 1$ STAT=Y PRED= 3.193 RANGE=0.258 WIDTH(Y)=1.032 RESP(Y)=1.0000 HSQC=C-2'

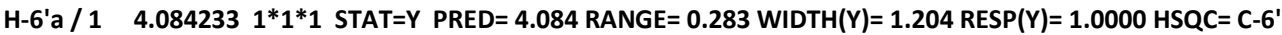
$\mathrm{H}-6$ 'b $/ 13.7350571 * 1 * 1$ STAT $=Y$ PRED $=3.734$ RANGE $=0.263$ WIDTH $(Y)=1.309$ RESP(Y)=1.0000 HSQC $=\mathrm{C}-6^{\prime}$ H-1" / 1 4.324156 1*1*1 STAT=Y PRED= 4.323 RANGE=0.273 WIDTH(Y)=0.961 RESP(Y)=1.0000 HSQC=C-1" H-5"a/ $13.8680981 * 1 * 1$ STAT=Y PRED= 3.868 RANGE= 0.333 WIDTH(Y)=1.135 RESP(Y)=1.0000 HSQC=C-5" H-5"b/ $13.5400421 * 1 * 1$ STAT=Y PRED= 3.538 RANGE=0.268 WIDTH(Y)=1.105 RESP(Y)=1.0000 HSQC=C-5" H-4" / 13.808179 1*1*1 STAT=Y PRED= 3.807 RANGE= 0.253 WIDTH(Y)=1.376 RESP(Y)=1.0000 HSQC=C-4" H-3" / $13.5292361{ }^{*} 1 * 1$ STAT=Y PRED= 3.527 RANGE= 0.273 WIDTH $(\mathrm{Y})=1.095$ RESP(Y)=1.0000 HSQC= C-3" H-2" / 13.594541 1*1*1 STAT=Y PRED= 3.596 RANGE=0.243 WIDTH(Y)=1.013 RESP(Y)=1.0000 HSQC $=\mathrm{C}-2 "$ H-1 / 1 2.245299 $1 * 1 * 1$ STAT=Y PRED= 2.244 RANGE $=0.313$ WIDTH $(\mathrm{Y})=1.154$ RESP $(\mathrm{Y})=1.0000 \mathrm{HSQC}=\mathrm{C}-1$ H-5 / $12.0864201 * 1 * 1$ STAT=Y PRED= 2.085 RANGE= 0.148 WIDTH(Y)=1.440 RESP(Y)=1.0000 HSQC $=\mathrm{C}-5$ H-4a / $12.3296121 * 1 * 1$ STAT=Y PRED= 2.336 RANGE= 0.168 WIDTH(Y)=1.505 RESP(Y)=1.0000 HSQC=C-4 $\mathrm{H}-4 \mathrm{~b} / 12.2608871 * 1 * 1$ STAT=Y PRED= 2.250 RANGE= $0.168 \mathrm{WIDTH}(\mathrm{Y})=1.596 \mathrm{RESP}(\mathrm{Y})=1.0000 \mathrm{HSQC}=\mathrm{C}-4$ $\mathrm{H}-3$ / $15.5980871 * 1 * 1$ STAT=Y PRED= 5.598 RANGE $=0.508$ WIDTH(Y)=1.216 RESP(Y)=1.0000 HSQC $=\mathrm{C}-3$ H-8 / $10.8529331 * 1 * 3$ STAT $=Y$ PRED $=0.852$ RANGE $=0.153$ WIDTH $(\mathrm{Y})=0.854$ RESP $(\mathrm{Y})=1.0000 \mathrm{HSQC}=\mathrm{C}-8$ $\mathrm{H}-9$ / $11.3068561 * 1 * 3$ STAT=Y PRED= 1.306 RANGE $=0.148$ WIDTH $(\mathrm{Y})=0.846$ RESP(Y)=1.0000 HSQC $=\mathrm{C}-9$ $\mathrm{H}-6 \mathrm{~b} / 11.1984231 * 1 * 1$ STAT=Y PRED= 1.198 RANGE $=0.173$ WIDTH $(\mathrm{Y})=0.940$ RESP $(\mathrm{Y})=1.0000 \mathrm{HSQC}=\mathrm{C}-6$ H-6a / $12.4248171 * 1 * 1$ STAT=Y PRED= 2.424 RANGE=0.148 WIDTH(Y)=1.076 RESP(Y)=1.0000 HSQC= C-6 MEOD CH $2 * S P I N=1$ SPECIES $=1 \mathrm{H}$ POPULATION $(\mathrm{Y})=0.31065$

MEOD / $23.3101761 * 1 * 1$ STAT $=Y$ PRED $=3.310$ RANGE $=0.048$ WIDTH $(\mathrm{Y})=0.704$ RESP(Y) $=1.0000$ MEOD_D 2*SPIN $=2$ SPECIES $=2 D$ POPULATION $(Y)=0.31065$

MEOD1 $/ 2-100.0000001 * 1 * 1$ STAT=Y PRED $=-99.999$ RANGE $=0.050$ WIDTH $(\mathrm{Y})=1.500 \operatorname{RESP}(\mathrm{Y})=1.0000$ MEOD2 / $2-100.0000001 * 1 * 1$ STAT=Y PRED $=-99.999$ RANGE $=0.050$ WIDTH(Y)=1.500 RESP(Y) $=1.0000$ WATER_H $2 * S P I N=1$ SPECIES $=1 \mathrm{H}$ POPULATION $(\mathrm{Y})=0.61710$

WATER $/ 3 \quad 4.8698102 * 1 * 1$ STAT $=Y$ PRED $=4.869$ RANGE $=0.048$ WIDTH $(Y)=1.142$ RESP $(Y)=1.0000$

COUPLING CONSTANTS(HZ):

J32_33 -12.3253 J H-10a H-10b STAT $=Y$ PRED $=-13.036$ RANGE $=2.190$

J32_55 1.8879 J H-10a H-4a STAT=Y PRED $=1.718$ RANGE $=2.190$

J32 562.2977 J H-10a H-4b STAT $=Y$ PRED $=2.341$ RANGE $=2.190$

J32_57 -1.6661 J H-10a H-3 STAT=Y PRED $=-1.032$ RANGE $=1.000$

J33_55 1.4655 J H-10b H-4a STAT=Y PRED= 1.411 RANGE $=1.000$

J33_56 -1.6592 J H-10b H-4b STAT $=Y$ PRED $=-1.662$ RANGE $=1.000$

J33_57 -1.2099 J H-10b H-3 STAT=Y PRED $=-1.043$ RANGE $=1.000$

J34_38 7.8700 J H-1' H-2' STAT=Y PRED $=7.767$ RANGE $=2.000$

J35_36 9.6663 J H-5' H-4' STAT=Y PRED= 9.797 RANGE $=2.000$

J35_39 2.3499 J H-5' H-6'a STAT=Y PRED= 6.276 RANGE= 2.040

J35_40 5.6179 J H-5' H-6'b STAT=Y PRED $=4.088$ RANGE $=2.990$

J36_37 8.7018 J H-4' H-3' STAT $=Y$ PRED $=8.983$ RANGE $=2.000$

J37_38 9.2537 J H-3' H-2' STAT=Y PRED= 9.335 RANGE $=2.000$

J39_40 -11.3935 J H-6'a H-6'b STAT=Y PRED= -11.205 RANGE $=1.000$

J4146 6.8608 J H-1" H-2" STAT=Y PRED = 7.759 RANGE $=2.000$

J42_43 -12.4600 J H-5"a H-5"b STAT=Y PRED $=-11.713$ RANGE $=1.000$

J42 443.2548 J H-5"a H-4" STAT $=Y$ PRED $=3.573$ RANGE $=2.040$

J43_44 1.8019 J H-5"b H-4" STAT=Y PRED= 3.168 RANGE= 2.180

J44 453.4685 J H-4" H-3" STAT=Y PRED= 3.169 RANGE= 2.000

J45_46 8.7500 J H-3" H-2" STAT=Y PRED= 9.535 RANGE= 2.000

J53_54 5.8406 J H-1 H-5 STAT $=Y$ PRED $=5.962$ RANGE $=2.550$ 


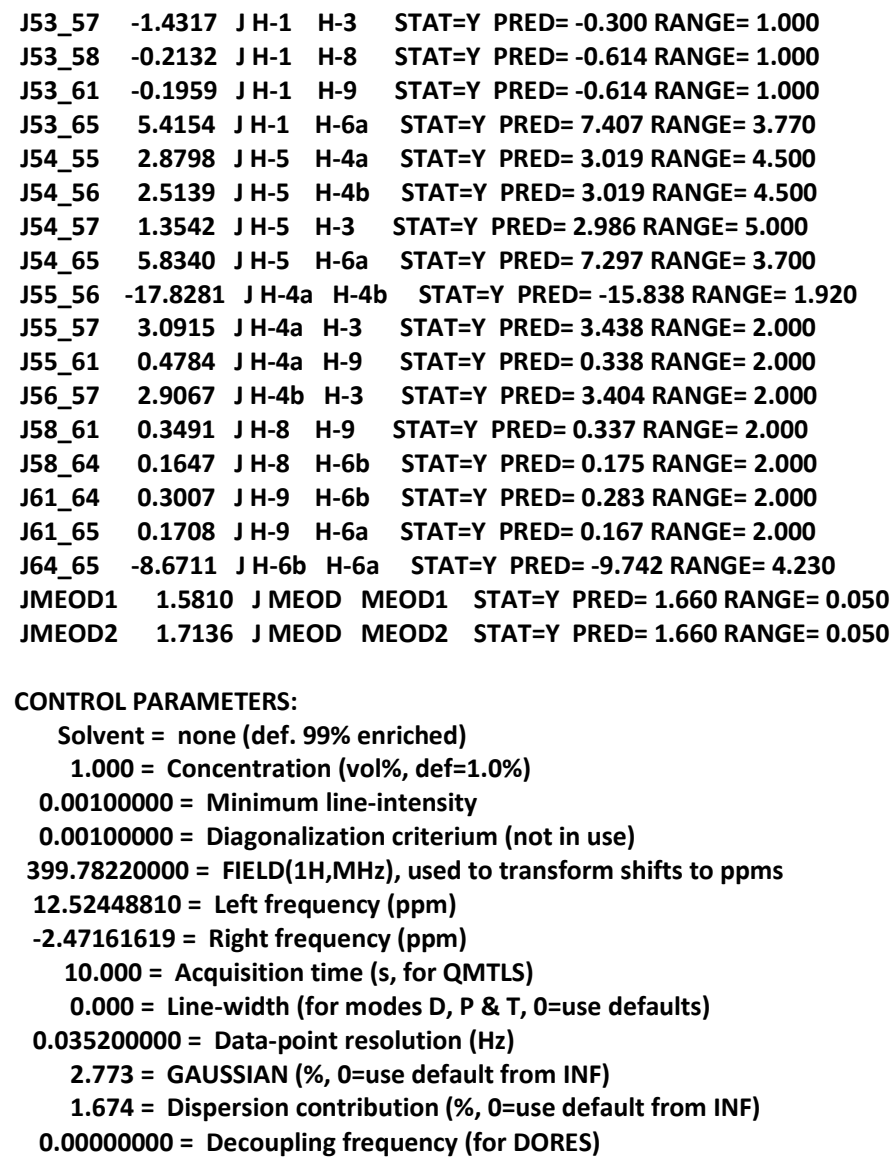

END of FILE

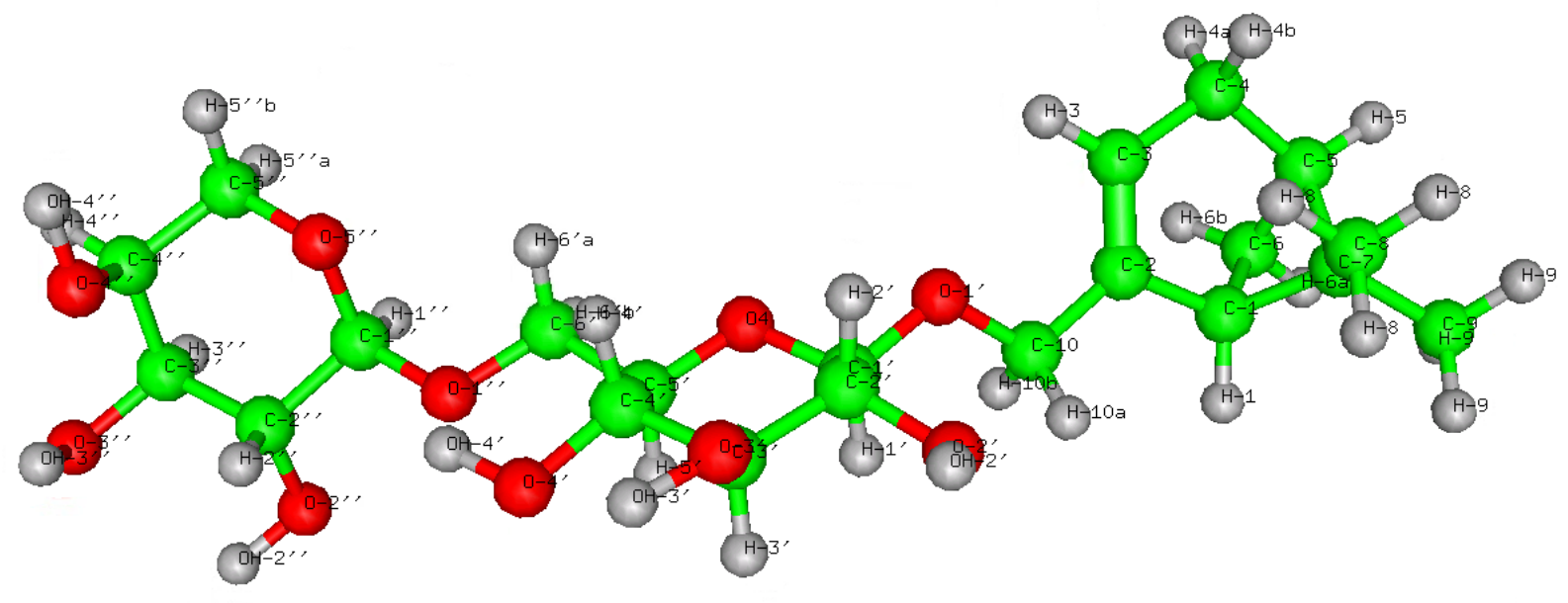




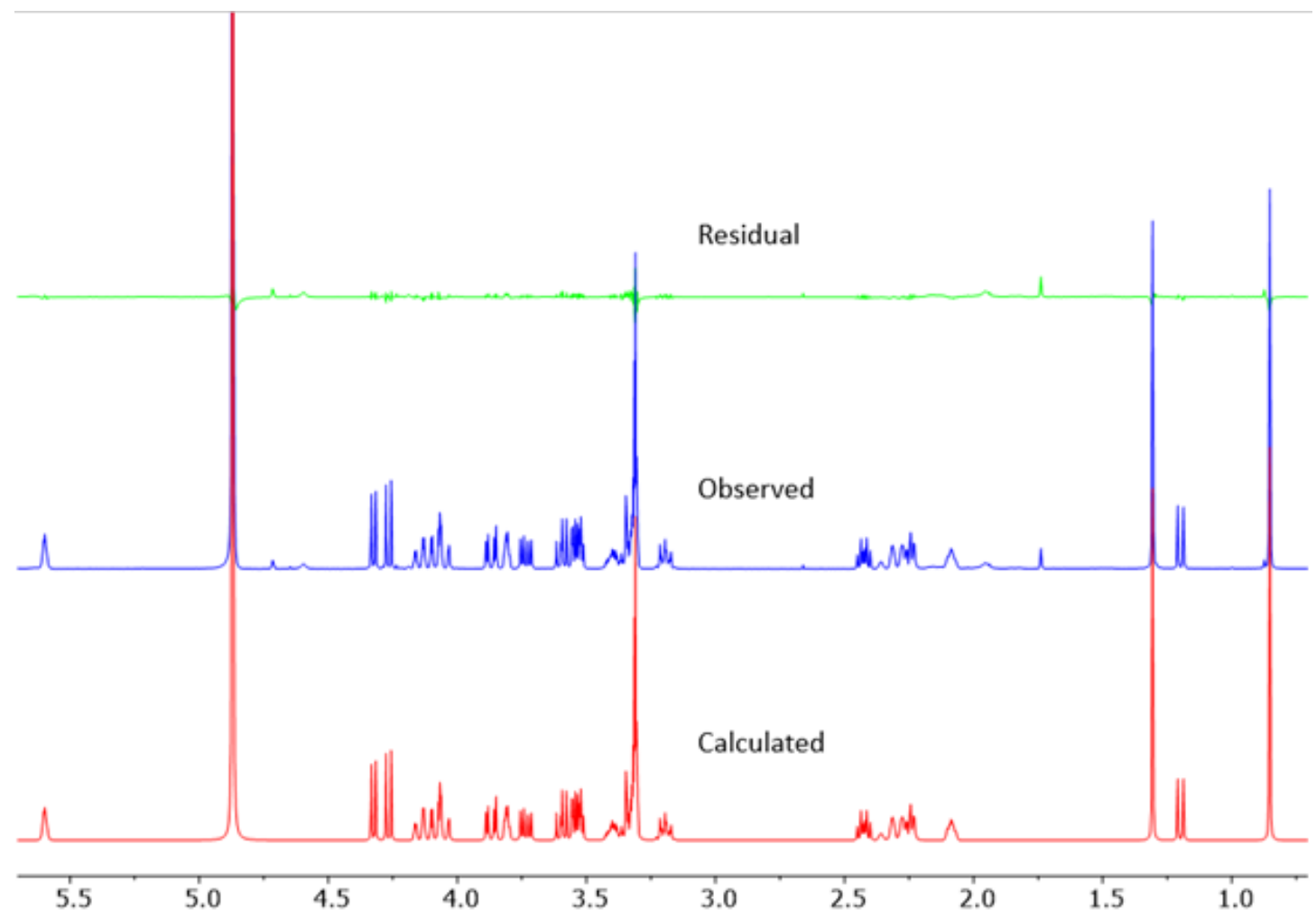

Figure S-7. The ${ }^{1} \mathrm{H}$ NMR fingerprint of 1 in $\mathrm{CD}_{3} \mathrm{OD}(400 \mathrm{MHz}, 298 \mathrm{~K})$ generated by PERCH

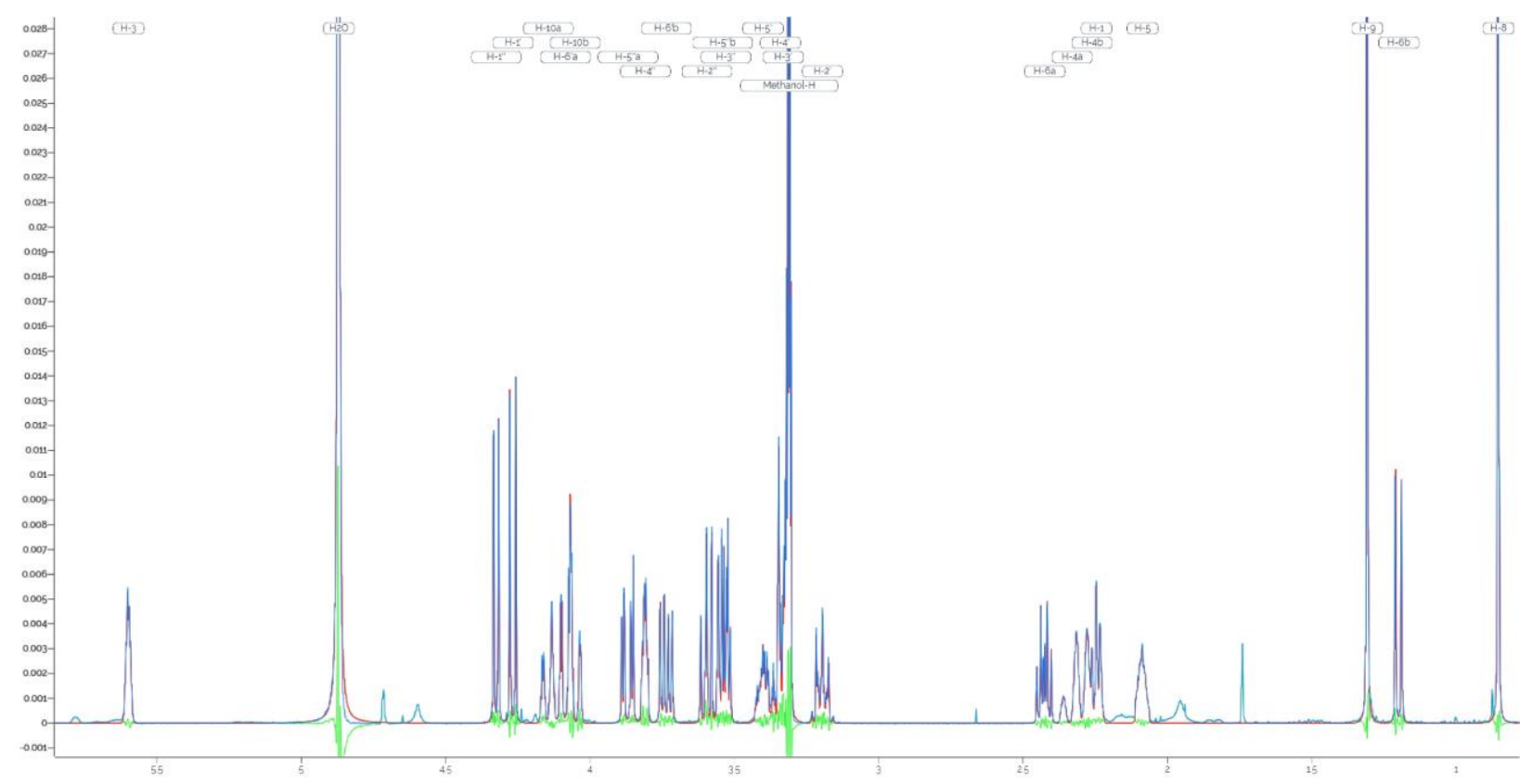

Figure S-8. The ${ }^{1} \mathrm{H}$ NMR fingerprint of 1 in $\mathrm{CD}_{3} \mathrm{OD}(400 \mathrm{MHz}, 298 \mathrm{~K})$ generated by $\mathrm{CT}$ 


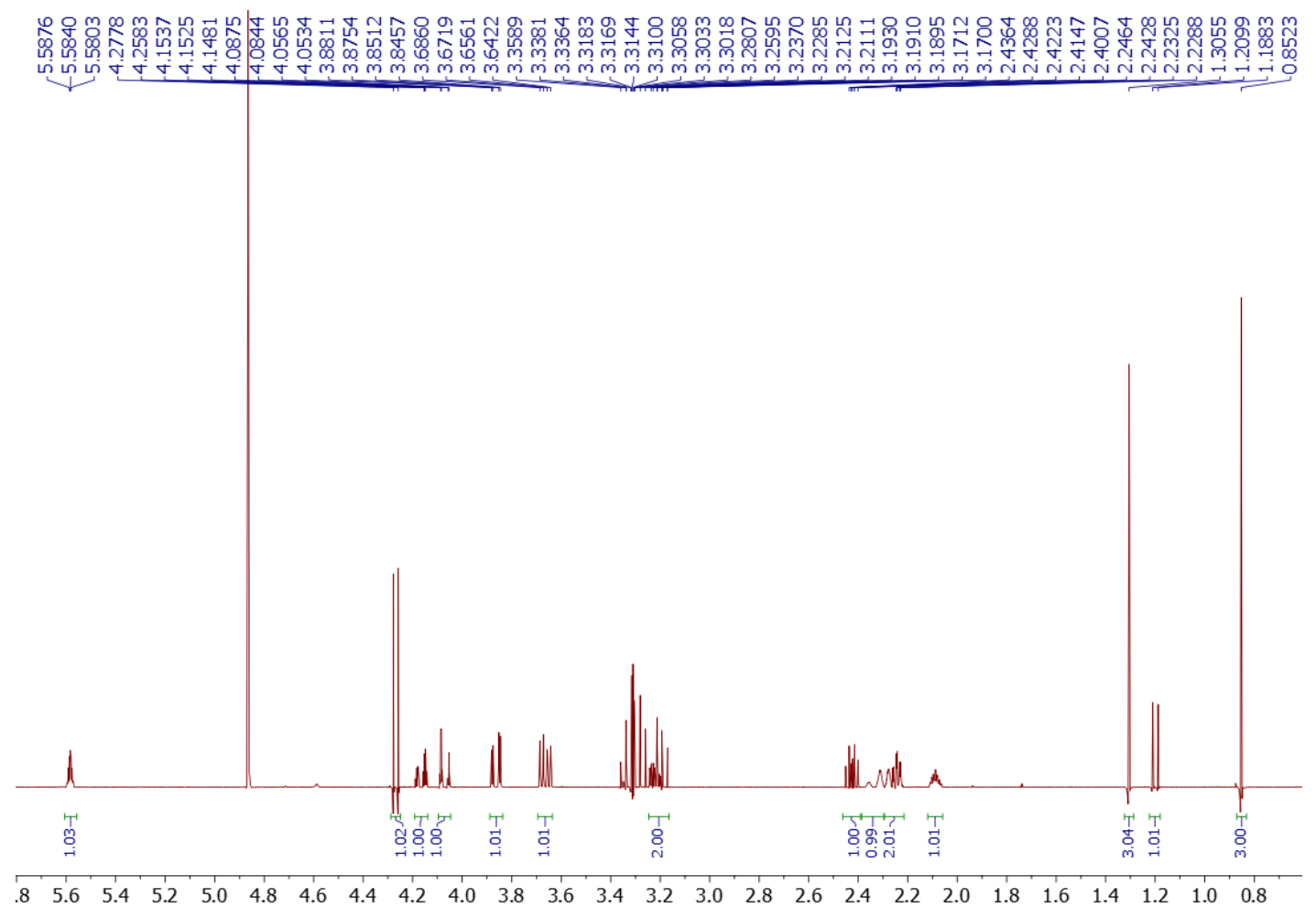

Figure S-9. ${ }^{1} \mathrm{H}$ NMR spectrum of 2 (400 MHz, $298 \mathrm{~K}$ ) in $\mathrm{CD}_{3} \mathrm{OD}$
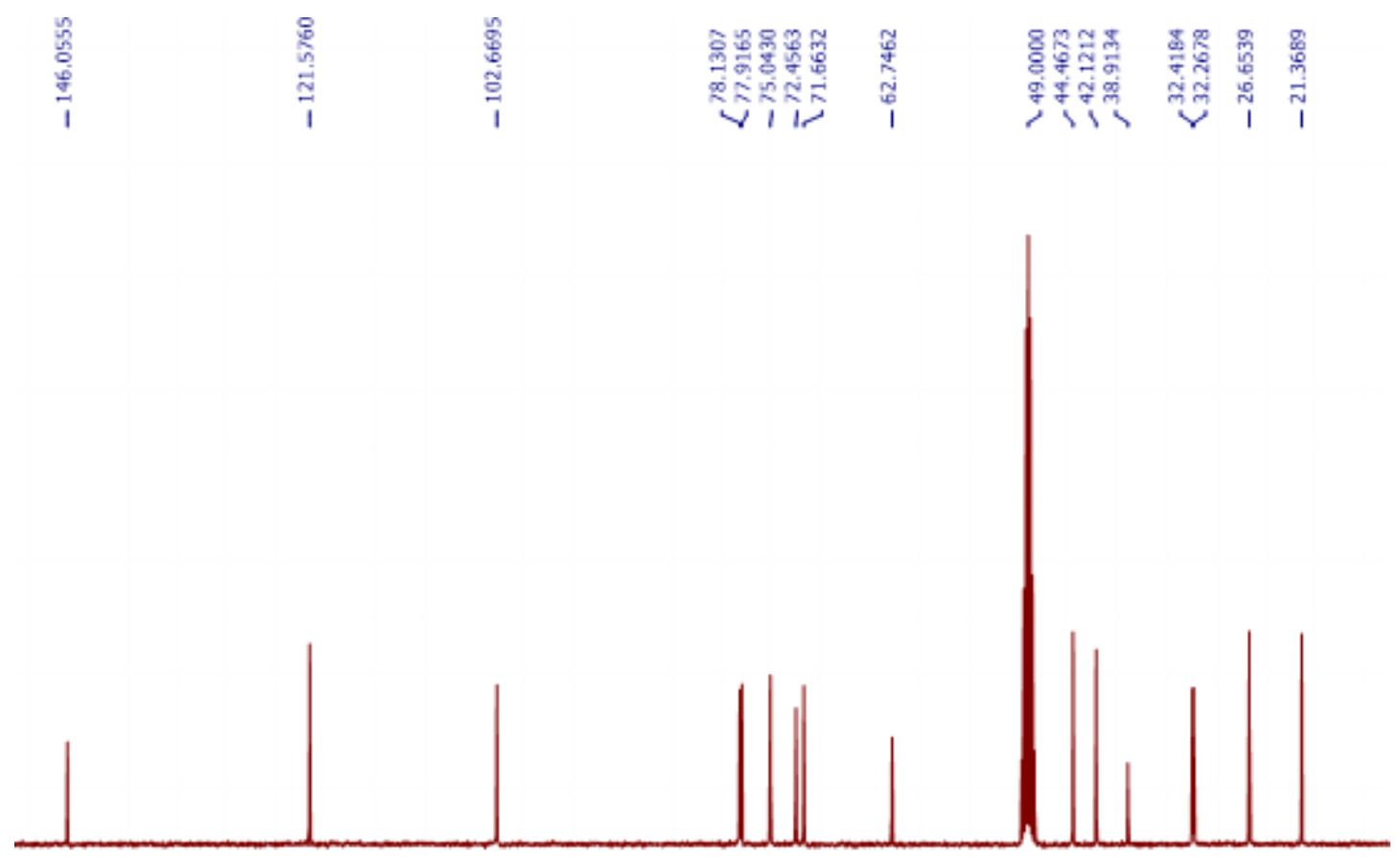

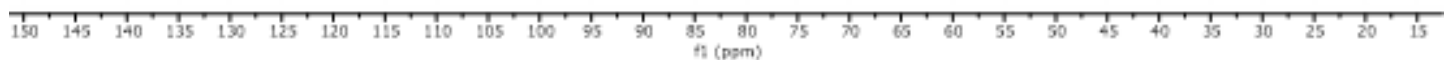

Figure S-10. ${ }^{13} \mathrm{C}$ NMR spectrum of 2 (100 MHz, $\left.298 \mathrm{~K}\right)$ in $\mathrm{CD}_{3} \mathrm{OD}$ 


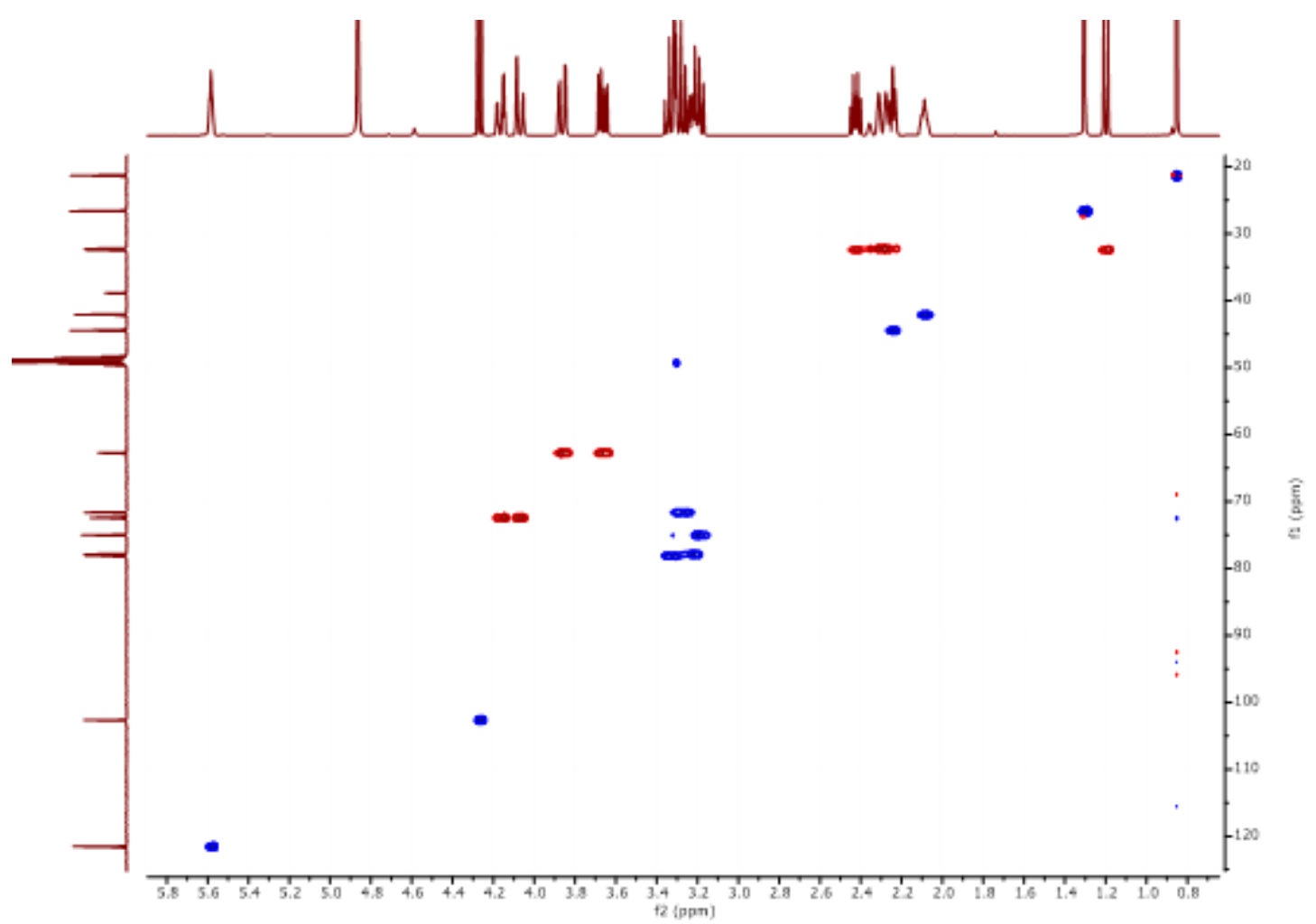

Figure S-11. HSQC spectrum of 2 (400 MHz, $298 \mathrm{~K}$ ) in $\mathrm{CD}_{3} \mathrm{OD}$

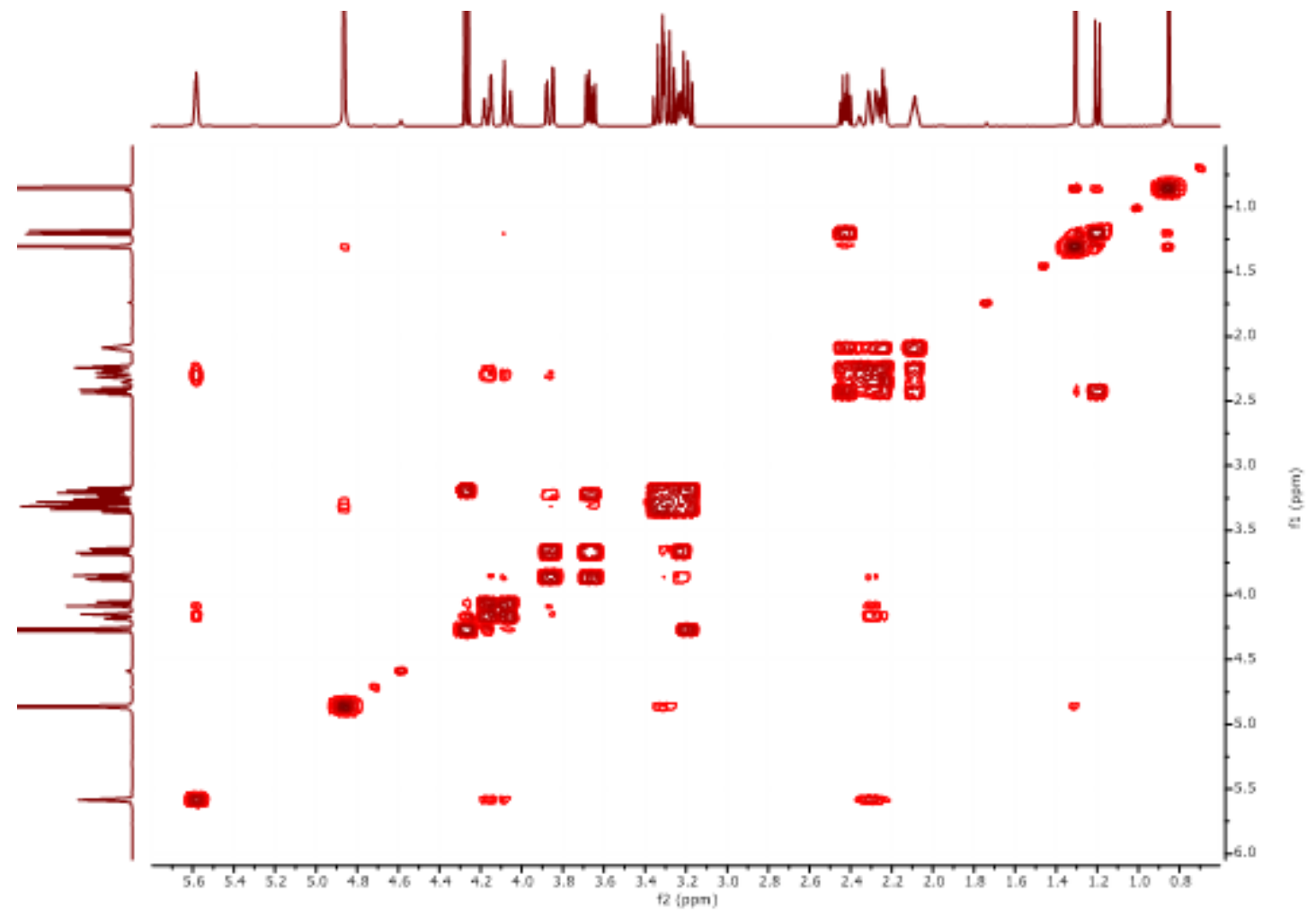

Figure S-12. COSY spectrum of $2(400 \mathrm{MHz}, 298 \mathrm{~K})$ in $\mathrm{CD}_{3} \mathrm{OD}$ 


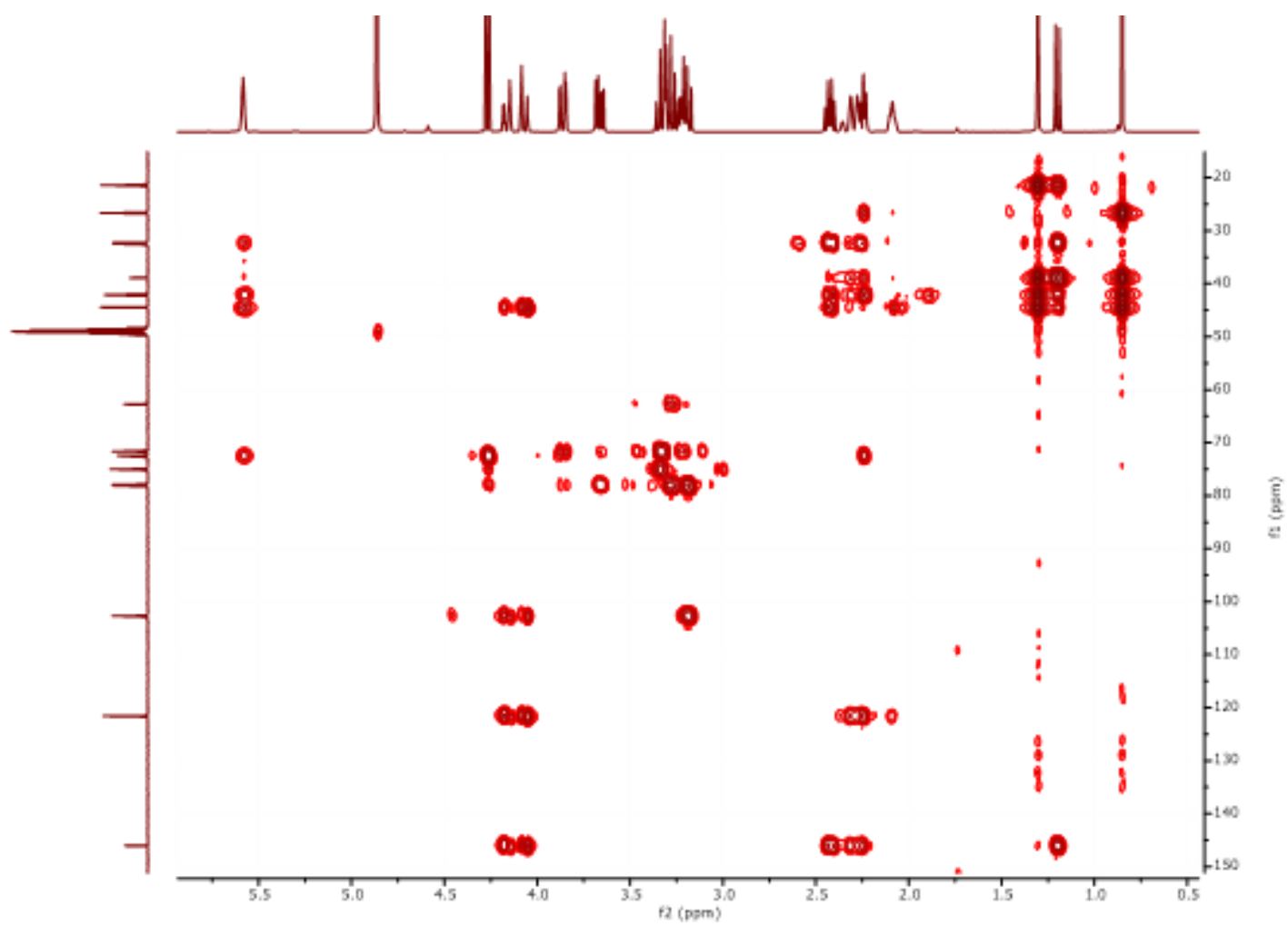

Figure S-13. $\mathrm{HMBC}$ spectrum of $2(400 \mathrm{MHz}, 298 \mathrm{~K})$ in $\mathrm{CD}_{3} \mathrm{OD}$ 


\section{Complete HiFSA Profile of Compound 2}

\footnotetext{
* NEW: the lines beginning by * are comment lines !

* To keep all the chemical shifts fixed during iteration

* replace "CHEMICAL SHIFTS(HZ):" by "..SHIFTS(HZ): fixed"

* The couplings can be fixed in the same way
}

ACTIVE SPECIES:1H

CHEMICAL SHIFTS(PPM):

PROTON $2 *$ SPIN $=1$ SPECIES $=1 \mathrm{H} \quad$ POPULATION $(\mathrm{Y})=0.22460$

$\mathrm{H}-10 \mathrm{a} / 14.1635331 * 1 * 1$ STAT=Y PRED 14.164 RANGE $=0.383$ WIDTH $(\mathrm{Y})=0.923$ RESP $(\mathrm{Y})=1.0000 \mathrm{HSQC}=\mathrm{C}-10$

$\mathrm{H}-10 \mathrm{~b} / 14.0730131{ }^{*} 1 * 1$ STAT=Y PRED $=4.073$ RANGE $=0.363$ WIDTH $(\mathrm{Y})=0.798$ RESP $(\mathrm{Y})=1.0000 \mathrm{HSQC}=\mathrm{C}-10$

H-1' / $14.2679801 * 1 * 1$ STAT=Y PRED= 4.268 RANGE $=0.283$ WIDTH(Y)=0.562 RESP(Y)=1.0000 HSQC $=\mathrm{C}-1^{\prime}$

H-5' / $13.2230901{ }^{*} 1^{*} 1$ STAT $=Y$ PRED $=3.223$ RANGE $=0.238$ WIDTH(Y)=0.894 RESP(Y)=1.0000 HSQC= C-5'

H-4' / $13.2816411 * 1 * 1$ STAT=Y PRED= 3.282 RANGE=0.213 WIDTH(Y)=0.361 RESP(Y)=0.6241 HSQC= C-4'

H-3' / $13.3340861 * 1 * 1$ STAT=Y PRED= 3.334 RANGE= 0.148 WIDTH(Y)=0.496 RESP(Y)=0.7206 HSQC=C-3'

H-2' / 1 3.192357 $1^{*}{ }^{*}{ }^{*} 1$ STAT $=Y$ PRED= 3.192 RANGE $=0.248$ WIDTH $(\mathrm{Y})=0.672$ RESP $(\mathrm{Y})=1.0000$ HSQC $=\mathrm{C}^{-2} \mathbf{2}^{\prime}$

H-6a'/ $13.8622441^{*} 1^{*} 1$ STAT=Y PRED= 3.862 RANGE= 0.173 WIDTH(Y)=1.001 RESP(Y)=1.0000 HSQC= C6'

H-6b'/ $13.6650621 * 1 * 1$ STAT=Y PRED= 3.665 RANGE= 0.188 WIDTH $(Y)=0.992$ RESP $(Y)=1.0000$ HSQC $=$ C6 $^{\prime}$

H-1 / 12.244731 1*1*1 STAT=Y PRED= 2.245 RANGE= 0.303 WIDTH $(\mathrm{Y})=0.760$ RESP(Y)=1.0000 HSQC= C-1

H-5 / $12.0880951 * 1 * 1$ STAT=Y PRED= 2.089 RANGE= 0.148 WIDTH(Y)=1.216 RESP $(\mathrm{Y})=1.0000$ HSQC $=\mathrm{C}-5$

H-4a / $12.3276871 * 1 * 1$ STAT=Y PRED= 2.328 RANGE= 0.153 WIDTH(Y)= 1.098 RESP(Y)=1.0000 HSQC $=$ C-4

$\mathrm{H}-4 \mathrm{~b} / 12.2615671 * 1 * 1$ STAT=Y PRED= 2.262 RANGE= 0.158 WIDTH(Y)=1.182 RESP(Y)=1.0000 HSQC= C-4

H-3 / $15.5840021 * 1 * 1$ STAT=Y PRED= 5.584 RANGE $=0.418$ WIDTH $(Y)=0.860$ RESP $(Y)=1.0000$ HSQC $=C-3$

H-8 / $10.8523911 * 1 * 3$ STAT $=Y$ PRED $=0.852$ RANGE $=0.198$ WIDTH $(Y)=0.493$ RESP(Y)=1.0000 HSQC=C-8

H-9 / $11.3055701 * 1 * 3$ STAT=Y PRED= 1.306 RANGE= 0.148 WIDTH(Y)=0.496 RESP $(\mathrm{Y})=1.0000$ HSQC $=\mathrm{C}-9$

H-6b / $1.1991681 * 1 * 1$ STAT=Y PRED= 1.199 RANGE= 0.163 WIDTH(Y)=0.552 RESP(Y)=1.0000 HSQC $=\mathrm{C}-6$

H-6a / $12.4255981 * 1 * 1$ STAT=Y PRED= 2.425 RANGE= 0.148 WIDTH(Y)=0.756 RESP(Y)=1.0000 HSQC= C-6

MEOD_CH 2*SPIN $=1$ SPECIES $=1 \mathrm{H}$ POPULATION $(\mathrm{Y})=0.23540$

MEOD $/ 23.3100281 * 1 * 1$ STAT=Y PRED $=3.310$ RANGE $=0.048 \operatorname{WIDTH}(\mathrm{Y})=0.657 \operatorname{RESP}(\mathrm{Y})=1.0000$

MEOD_D 2*SPIN $=2$ SPECIES $=2 D$ POPULATION $(Y)=0.23540$

MEOD1/ $2-100.0000001 * 1 * 1$ STAT=Y PRED $=-99.999 \operatorname{RANGE}=0.050 \operatorname{WIDTH}(\mathrm{N})=1.500 \operatorname{RESP}(\mathrm{Y})=1.0000$

MEOD2/ $2-100.0000001 * 1 * 1$ STAT=Y PRED $=-99.999$ RANGE $=0.050 \operatorname{WIDTH}(\mathrm{N})=1.500 \operatorname{RESP}(\mathrm{Y})=1.0000$

WATER_H $2 *$ SPIN $=1$ SPECIES $=1 \mathrm{H} \quad$ POPULATION $(Y)=0.54000$

WATER/ $3 \quad 4.8647312 * 1 * 1$ STAT=Y PRED $=4.865$ RANGE $=0.448 \operatorname{WIDTH}(\mathrm{Y})=1.124 \operatorname{RESP}(\mathrm{Y})=1.0000$

COUPLING CONSTANTS(HZ):

J23_24 -12.3915 J H-10a H-10b STAT=Y PRED $=-13.037$ RANGE $=2.190$

J23_38 1.8734 J H-10a H-4a STAT=Y PRED $=1.813$ RANGE $=1.000$

J23_39 2.3937 J H-10a H-4b STAT $=Y$ PRED $=2.490$ RANGE $=1.000$

J23_40 -1.7276 J H-10a H-3 STAT=Y PRED $=-1.033$ RANGE $=1.000$

J24_38 1.4180 J H-10b H-4a STAT $=Y$ PRED $=1.464$ RANGE $=1.000$

J24_39 -1.7146 J H-10b H-4b STAT=Y PRED $=-1.736$ RANGE $=1.000$

J24_40 -1.1284 J H-10b H-3 STAT=Y PRED $=-0.862$ RANGE $=1.000$

J25_29 7.8494 J H-1' H-2' STAT=Y PRED $=7.758$ RANGE $=2.000$

J26_27 9.7866 J H-5' H-4' STAT=Y PRED= 9.759 RANGE= 2.000

J26_28 -0.3325 J H-5' H-3' STAT $=Y$ PRED $=-0.301$ RANGE $=2.000$

J26_30 2.3131 J H-5' H-6a' STAT=Y PRED= 3.660 RANGE= 2.560

J26_31 5.8967 J H-5' H-6b' STAT=Y PRED= 3.919 RANGE $=2.690$

J27_28 9.0154 J H-4' H-3' STAT $=Y$ PRED $=8.966$ RANGE $=2.000$

J28 299.2633 J H-3' H-2' STAT=Y PRED= 9.310 RANGE $=2.000$

J30_31 -11.9171 J H-6a' H-6b' STAT=Y PRED $=-12.099$ RANGE $=1.000$

J36_37 5.8621 J H-1 H-5 STAT=Y PRED = 5.960 RANGE= 2.550

J36_40 -1.4042 J H-1 H-3 STAT $=Y$ PRED $=-0.300$ RANGE $=1.000$

J36_41 -0.2003 J H-1 H-8 STAT $=Y$ PRED $=-0.210$ RANGE $=1.000$

J36_44 -0.2245 J H-1 H-9 STAT $=Y$ PRED $=-0.614$ RANGE $=1.000$

J36_48 5.4242 J H-1 H-6a STAT=Y PRED= 7.408 RANGE= 3.780 


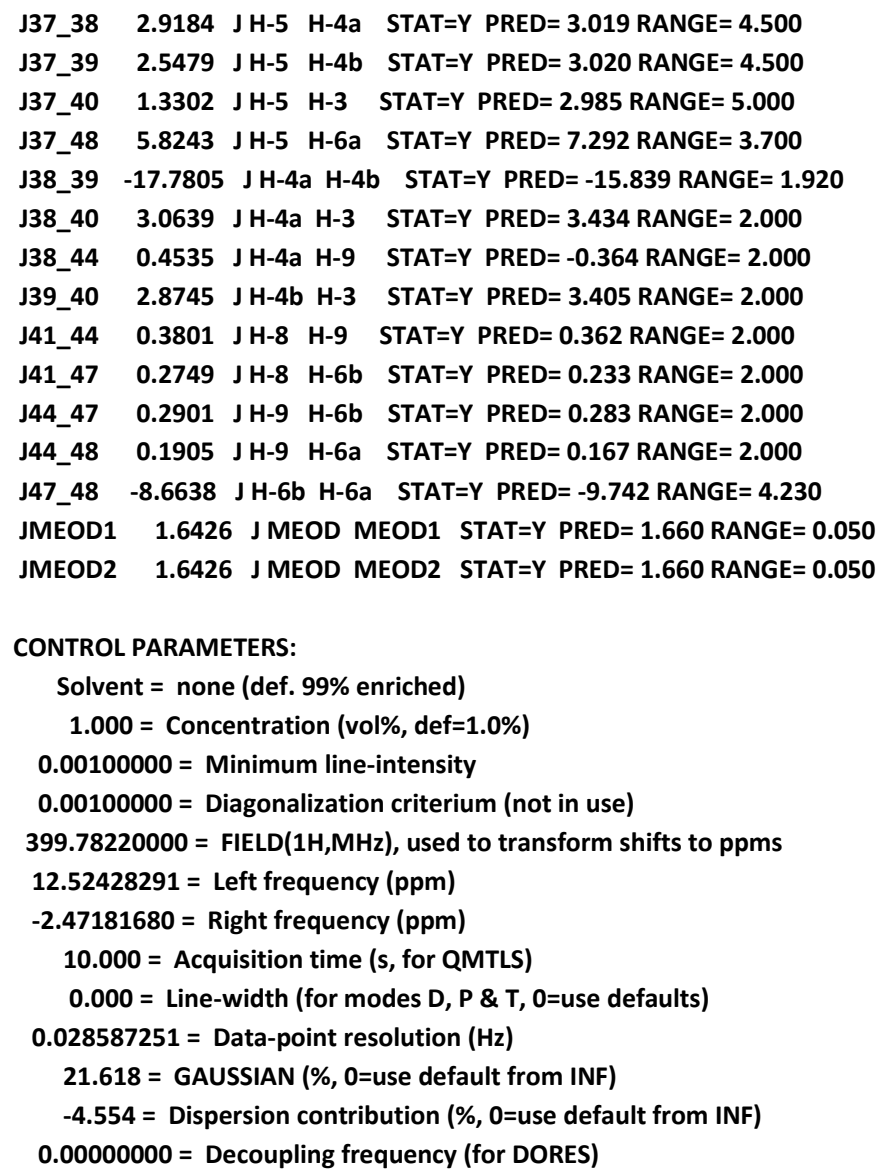

END of FILE

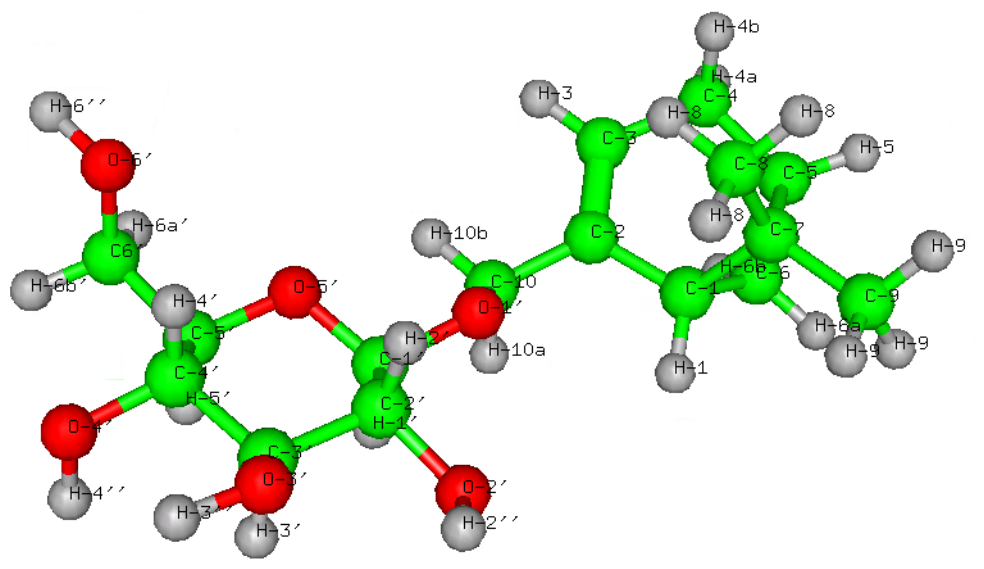




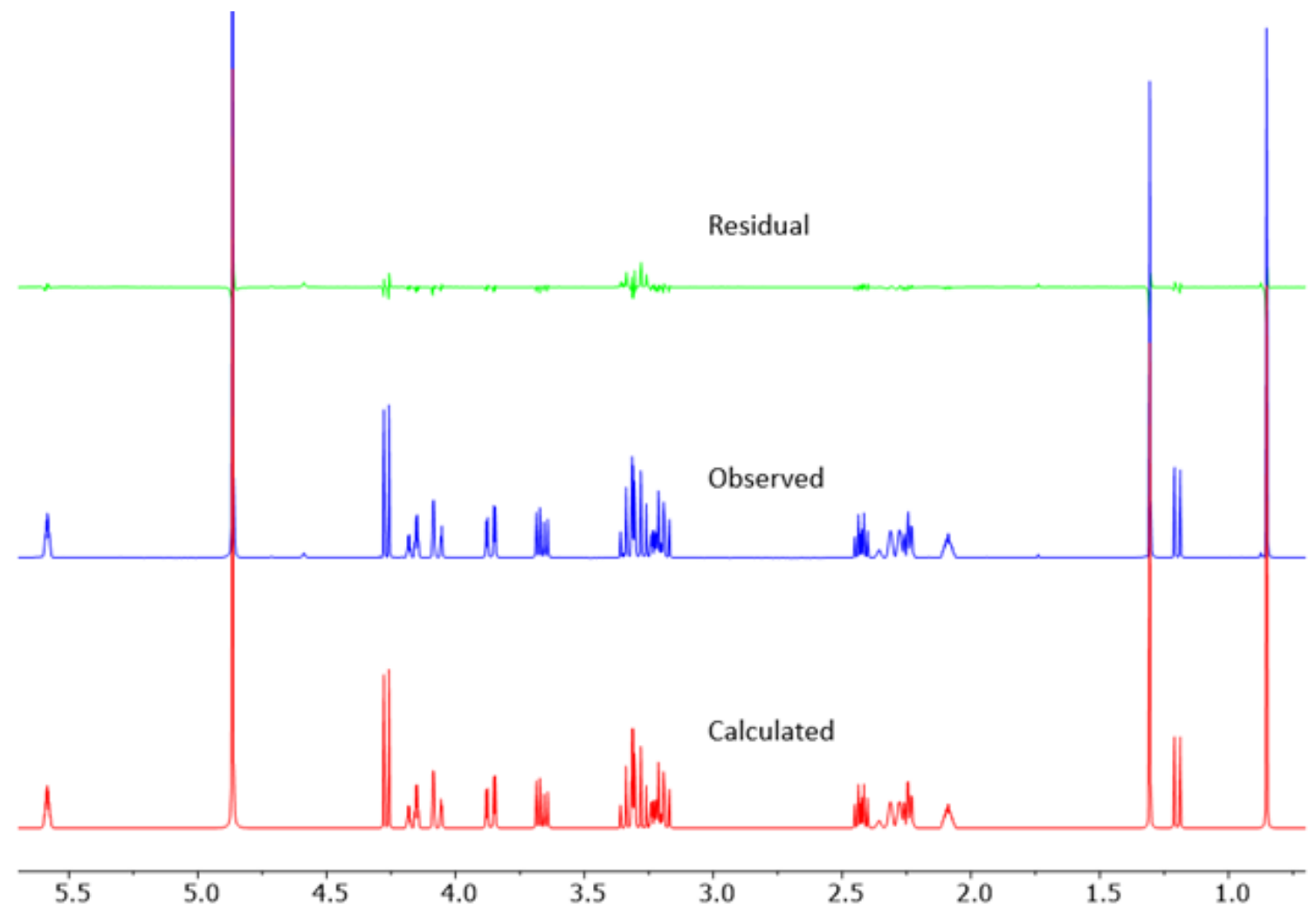

Figure S-14. The ${ }^{1} \mathrm{H}$ NMR fingerprint of 2 in $\mathrm{CD}_{3} \mathrm{OD}(400 \mathrm{MHz}, 298 \mathrm{~K})$ generated by PERCH 


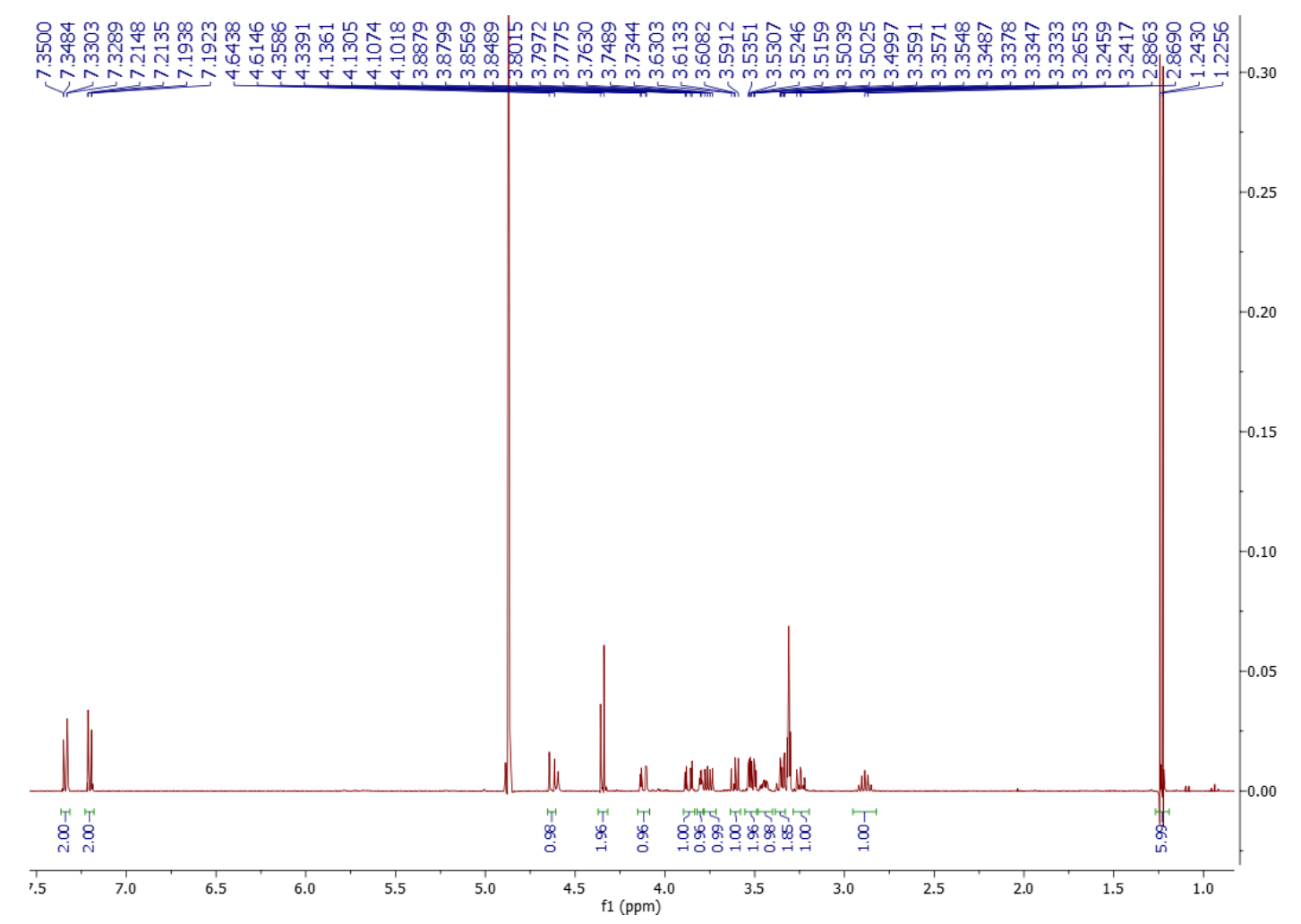

Figure S-15. ${ }^{1} \mathrm{H}$ NMR spectrum of 3 (400 MHz, $298 \mathrm{~K}$ ) in $\mathrm{CD}_{3} \mathrm{OD}$
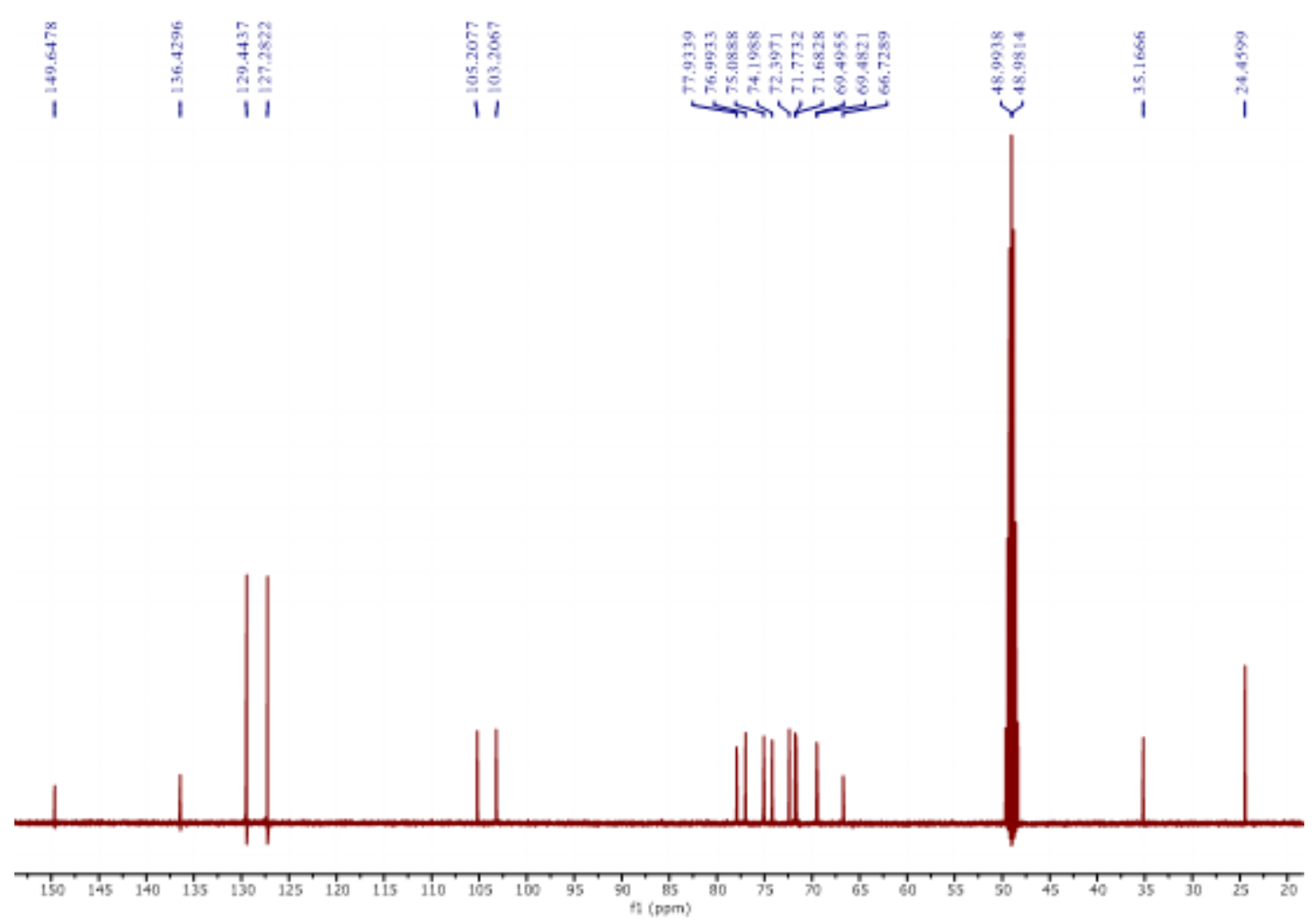

Figure S-16. ${ }^{13} \mathrm{C}$ NMR spectrum of $3(100 \mathrm{MHz}, 298 \mathrm{~K})$ in $\mathrm{CD}_{3} \mathrm{OD}$ 


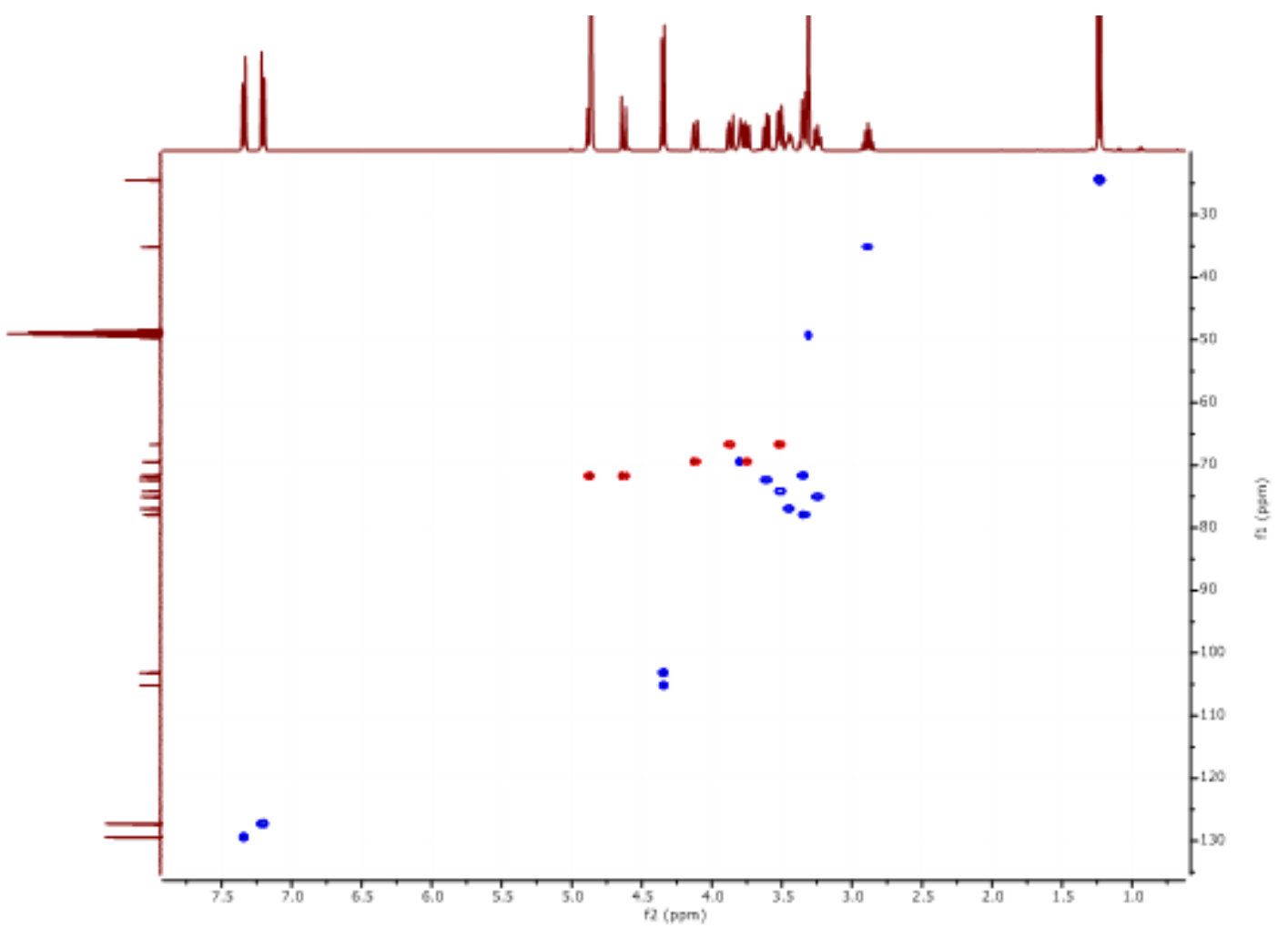

Figure S-17. HSQC spectrum of 3 (400 MHz, $298 \mathrm{~K}$ ) in $\mathrm{CD}_{3} \mathrm{OD}$

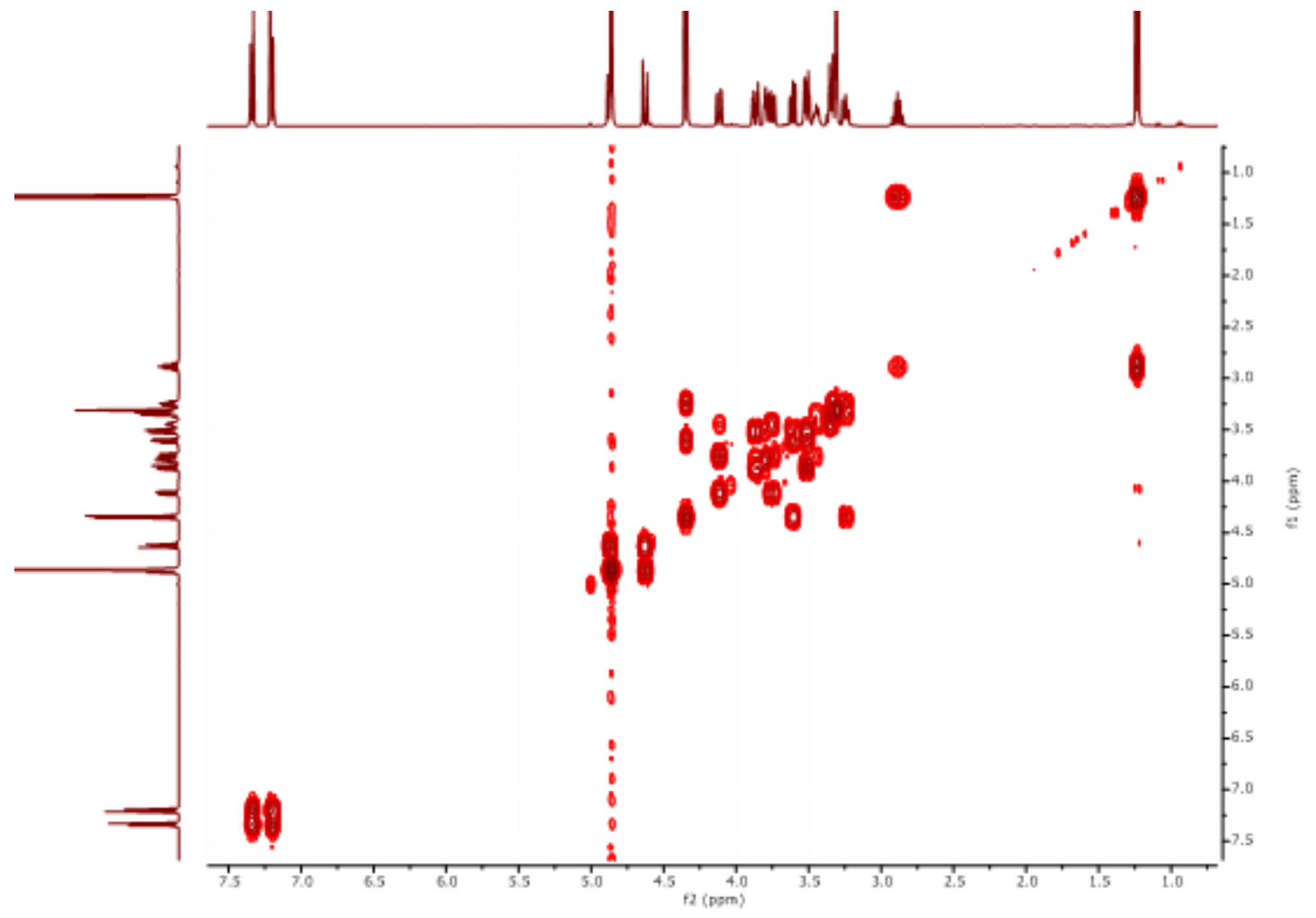

Figure S-18. COSY spectrum of $3(400 \mathrm{MHz}, 298 \mathrm{~K})$ in $\mathrm{CD}_{3} \mathrm{OD}$ 


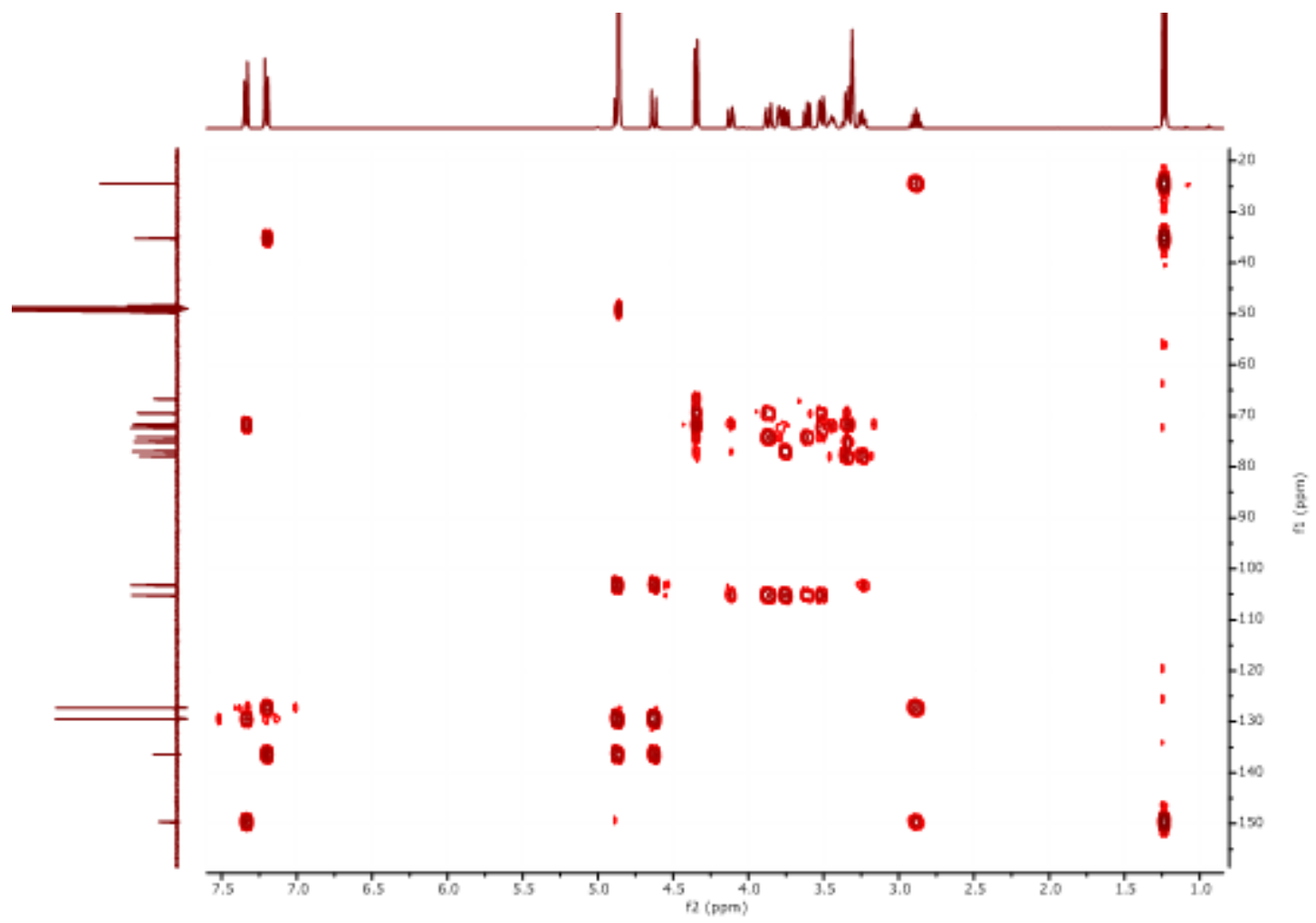

Figure S-19. $\mathrm{HMBC}$ spectrum of 3 (400 MHz, $298 \mathrm{~K}$ ) in $\mathrm{CD}_{3} \mathrm{OD}$ 


\section{Complete HiFSA Profile of Compound 3}

\footnotetext{
* NEW: the lines beginning by * are comment lines !

* To keep all the chemical shifts fixed during iteration

* replace "CHEMICAL SHIFTS(HZ):" by "..SHIFTS(HZ): fixed"

* The couplings can be fixed in the same way
}

CHEMICAL SHIFTS(PPM):

PROTON 2*SPIN $=1$ SPECIES $=1 \mathrm{H} \quad$ POPULATION $(\mathrm{Y})=0.05982$

H-1' / 1 4.348882 $1^{*} 1^{*} 1$ STAT=Y PRED= 4.349 RANGE=0.263 WIDTH(Y)=0.770 RESP(Y)=1.0000 HSQC= C-1' H-5' / $13.4484191 * 1 * 1$ STAT=Y PRED= 3.448 RANGE= 0.263 WIDTH(Y)=1.353 RESP(Y)=1.0000 HSQC $=C^{\prime}-5^{\prime}$ H-4' / $13.35175611^{*}{ }^{*} 1$ STAT=Y PRED= 3.352 RANGE= 0.308 WIDTH(Y)=1.084 RESP(Y)=1.0000 HSQC=C-4' H-3' / $13.3406551 * 1 * 1$ STAT=Y PRED= 3.341 RANGE= 0.173 WIDTH(Y)=1.003 RESP(Y)=1.0000 HSQC $=$ C-3' H-2' / $13.2454641{ }^{*} 1^{*} 1$ STAT=Y PRED= 3.245 RANGE= 0.208 WIDTH(Y)=1.014 RESP(Y)=1.0000 HSQC= C-2' H-6'a $/ 1$ 4.118474 $1 * 1 * 1$ STAT=Y PRED= 4.118 RANGE=0.278 WIDTH(Y)=1.252 RESP(Y)=1.0000 HSQC= C-6' H-6'b $/ 13.7564341 * 1 * 1$ STAT $=Y$ PRED $=3.756$ RANGE $=0.293$ WIDTH $(Y)=1.328$ RESP(Y)=1.0000 HSQC $=C-6{ }^{\prime}$ $\mathrm{H}-1 " / 1$ 4.347198 1*1*1 STAT=Y PRED= 4.347 RANGE=0.283 WIDTH(Y)=0.901 RESP(Y)=1.0000 HSQC=C-1" H-5"a/ $13.8675401 * 1 * 1$ STAT=Y PRED= 3.867 RANGE= 0.298 WIDTH(Y)=1.153 RESP(Y)=1.0000 HSQC=C5" $\mathrm{H}-5 " \mathrm{~b} / 13.5181581 * 1 * 1$ STAT $=Y$ PRED= 3.518 RANGE $=0.283$ WIDTH(Y)=1.081 RESP(Y)=1.0000 HSQC=C5" H-4" / $13.7996581{ }^{*} 1{ }^{*} 1$ STAT=Y PRED= 3.800 RANGE= 0.278 WIDTH(Y)=1.277 RESP(Y)=1.0000 HSQC=C-4" H-3" / $13.5106451 * 1 * 1$ STAT=Y PRED= 3.510 RANGE $=0.238$ WIDTH $(\mathrm{Y})=1.156$ RESP(Y)=1.0000 HSQC $=\mathrm{C}-3$ " H-2" / 13.609691 1*1*1 STAT=Y PRED= 3.610 RANGE= 0.233 WIDTH(Y)=1.030 RESP(Y)=1.0000 HSQC= C-2" H-3_5 / $17.2044231 * 2 * 1$ STAT=Y PRED= 7.204 RANGE $=0.228$ WIDTH $(\mathrm{Y})=0.697$ RESP(Y)=1.0000 HSQC=C-3_5 H-2_6 / $17.3388021 * 2 * 1$ STAT=Y PRED= 7.339 RANGE=0.148 WIDTH(Y)=0.655 RESP(Y)=1.0000 HSQC=C-2_6 H-8 / $12.886221 \quad 1 * 1 * 1$ STAT=Y PRED= 2.886 RANGE= 0.163 WIDTH(Y)=1.441 RESP(Y)=1.0000 HSQC=C-8 H-9 / $11.2343601 * 1 * 3$ STAT=Y PRED= 1.234 RANGE= 0.148 WIDTH $(Y)=0.437$ RESP(Y)=1.0000 HSQC $=$ C-9 H-7a / $14.8740001 * 1 * 1$ STAT=Y PRED= 4.873 RANGE $=0.358$ WIDTH $(Y)=1.028$ RESP(Y)=1.0000 HSQC=C-7 H-7b / $14.6301721 * 1 * 1$ STAT=Y PRED= 4.630 RANGE $=0.333$ WIDTH $(\mathrm{Y})=0.943$ RESP $(\mathrm{Y})=1.0000 \mathrm{HSQC}=\mathrm{C}-7$ $\mathrm{H}-10 / 11.2345581 * 1 * 3$ STAT=Y PRED $=1.234$ RANGE $=0.148$ WIDTH $(\mathrm{Y})=0.884$ RESP(Y)=1.0000 HSQC $=\mathrm{C}-10$ MEOD_CH 2*SPIN $=1$ SPECIES $=1 \mathrm{H} \quad$ POPULATION $(\mathrm{Y})=0.23249$ MEOD / $23.3101741^{*} 1 * 1$ STAT=Y PRED= 3.310 RANGE $=0.048$ WIDTH $(Y)=0.969 \operatorname{RESP}(\mathrm{Y})=1.0000$ MEOD_D $2 * S P I N=2$ SPECIES $=2 D \quad$ POPULATION $(Y)=0.23249$

MEOD1 / $2-100.0000001 * 1 * 1$ STAT=Y PRED $=-99.999$ RANGE $=0.050 \mathrm{WIDTH}(\mathrm{N})=1.500 \mathrm{RESP}(\mathrm{Y})=1.0000$ MEOD2 / $2-100.0000001 * 1 * 1$ STAT=Y PRED $=-99.999$ RANGE= 0.050 WIDTH(N) $=1.500$ RESP(Y) $=1.0000$ WATER_H 2*SPIN $=1$ SPECIES $=1 \mathrm{H} \quad$ POPULATION $(\mathrm{Y})=0.70769$ WATER / $3 \quad 4.8712202 * 1 * 1$ STAT $=Y$ PRED $=4.871$ RANGE $=0.048$ WIDTH $(Y)=1.963$ RESP(Y $)=1.0000$

COUPLING CONSTANTS(HZ):

J32_36 7.8510 J H-1' H-2' STAT=Y PRED $=7.758$ RANGE $=2.000$

J33_34 9.6805 JH-5' H-4' STAT $=Y$ PRED $=9.759$ RANGE $=2.000$

J33 37 2.2197 J H-5' H-6'a STAT $=$ Y PRED $=3.975$ RANGE $=3.000$

J33_38 5.9029 J H-5' H-6'b STAT=Y PRED $=4.082$ RANGE $=3.050$

J34_35 9.1379 J H-4' H-3' STAT=Y PRED $=8.983$ RANGE $=2.000$

J35_36 9.2140 J H-3' H-2' STAT=Y PRED= 9.331 RANGE= 2.000

J37_38 -11.4293 J H-6'a H-6'b STAT=Y PRED $=-9.168$ RANGE $=1.530$

J39_44 6.8611 J H-1" H-2" STAT=Y PRED= 7.758 RANGE= 2.000

J40_41 -12.4860 J H-5"a H-5"b STAT=Y PRED $=-11.713$ RANGE $=1.000$

J40_42 3.2037 J H-5"a H-4" STAT=Y PRED= 3.582 RANGE= 2.040

J41_42 1.8097 J H-5"b H-4" STAT=Y PRED= 3.167 RANGE= 2.180

J42_43 3.5046 J H-4" H-3" STAT=Y PRED= 3.175 RANGE= 2.000

J43_44 8.7989 J H-3" H-2" STAT=Y PRED= 9.535 RANGE= 2.000

J51_54 1.8454 J H-3_5 H-3_5 STAT $=Y$ PRED $=1.948$ RANGE $=1.000$

J51_52 7.8712 J H-3_5 H-2_6 STAT $=Y$ PRED $=7.861$ RANGE $=1.000$

J51_53 0.5663 J H-2_6 H-3_5 STAT $=Y$ PRED $=0.524$ RANGE $=1.000$

J54_55 -0.1800 J H-3_5 H-8 STAT $=Y$ PRED $=-0.559$ RANGE $=1.000$

J54_59 0.4860 J H-3_5 H-7a STAT $=Y$ PRED $=0.477$ RANGE $=1.000$

J54_60 0.2026 J H-3 5 H-7b STAT $=Y$ PRED $=0.475$ RANGE $=1.000$

J52_53 1.8783 J H-2_6 H-2_6 STAT $=Y$ PRED $=1.968$ RANGE $=1.000$

J52 $550.2833 \mathrm{JH}-26 \mathrm{H}-8$ STAT $=$ PRED $=0.288$ RANGE $=1.000$

J53_59 -0.3133 J H-2_6 H-7a STAT=Y PRED $=-0.500$ RANGE $=1.000$

J53_60 -0.5730 J H-2_6 H-7b STAT=Y PRED $=-0.530$ RANGE $=1.000$

J55_56 6.9351 J H-8 $\quad$ H-9 STAT $=Y$ PRED $=6.944$ RANGE $=1.000$

J55_61 6.9143 J H-8 H-10 STAT=Y PRED $=6.944$ RANGE $=1.000$

J59_60 -11.5105 J H-7a H-7b STAT=Y PRED= -13.128 RANGE $=2.190$

JMEOD1 1.6379 J MEOD MEOD1 STAT=Y PRED= 1.660 RANGE $=0.050$ 
JMEOD2 1.6379 J MEOD MEOD2 STAT $=Y$ PRED $=1.660$ RANGE $=0.050$

CONTROL PARAMETERS:

Solvent $=$ none (def. $99 \%$ enriched)

$1.000=$ Concentration $($ vol\%, def $=1.0 \%)$

$0.00100000=$ Minimum line-intensity

$0.00100000=$ Diagonalization criterium (not in use)

$399.78220000=\operatorname{FIELD}(1 \mathrm{H}, \mathrm{MHz})$, used to transform shifts to ppms

$12.52437940=$ Left frequency $(\mathrm{ppm})$

$-2.47171787=$ Right frequency $(\mathrm{ppm})$

$10.000=$ Acquisition time (s, for QMTLS)

$0.000=$ Line-width (for modes D, P \& T, $0=$ use defaults)

$0.028587251=$ Data-point resolution $(\mathrm{Hz})$

33.343 = GAUSSIAN (\%, $0=$ use default from INF)

$-9.931=$ Dispersion contribution (\%, $0=$ use default from INF)

$\mathbf{0 . 0 0 0 0 0 0 0 0}=$ Decoupling frequency (for DORES)

END of FILE

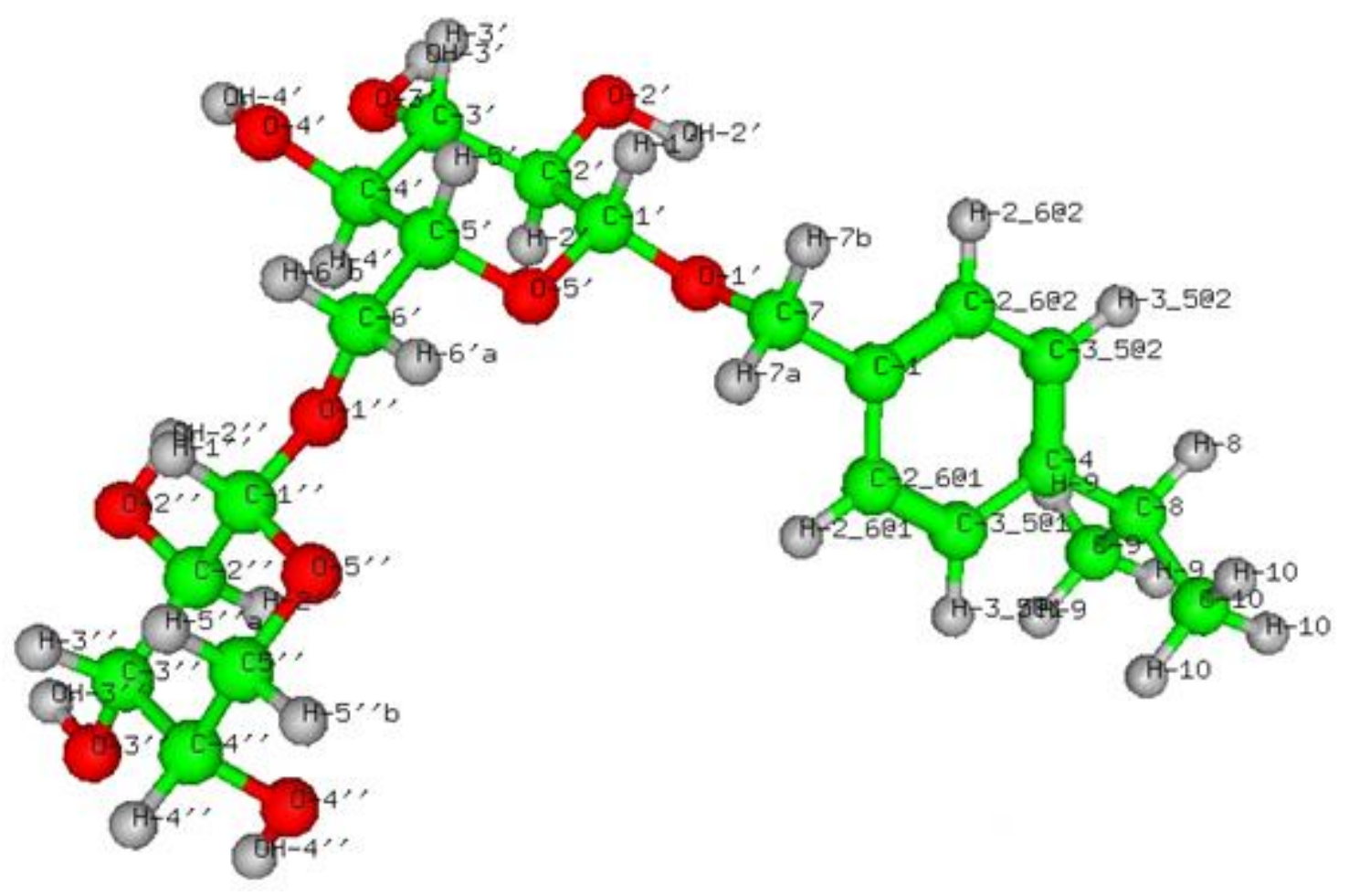




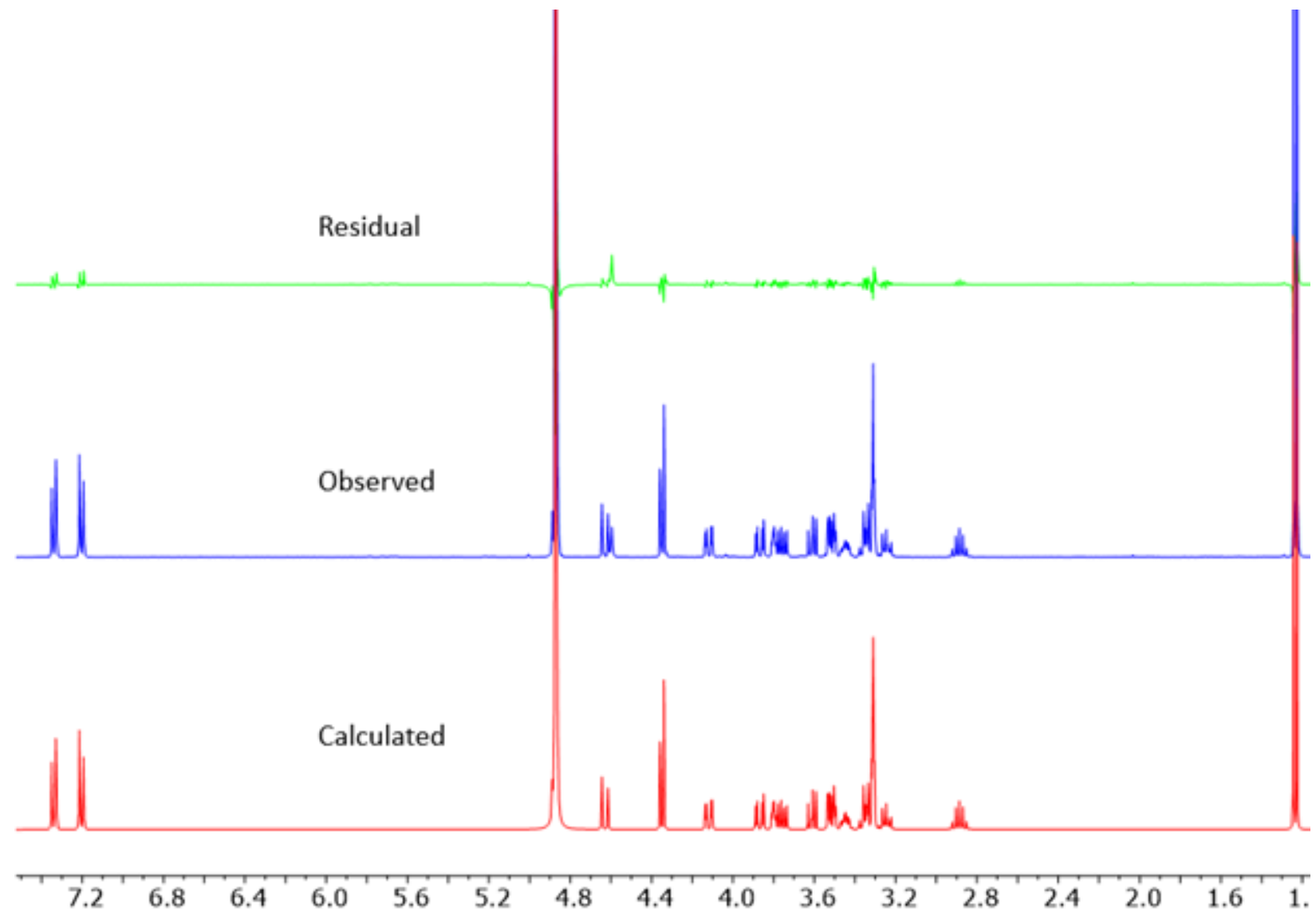

Figure S-20. The ${ }^{1} \mathrm{H}$ NMR fingerprint of 3 in $\mathrm{CD}_{3} \mathrm{OD}(400 \mathrm{MHz}, 298 \mathrm{~K})$ generated by PERCH 


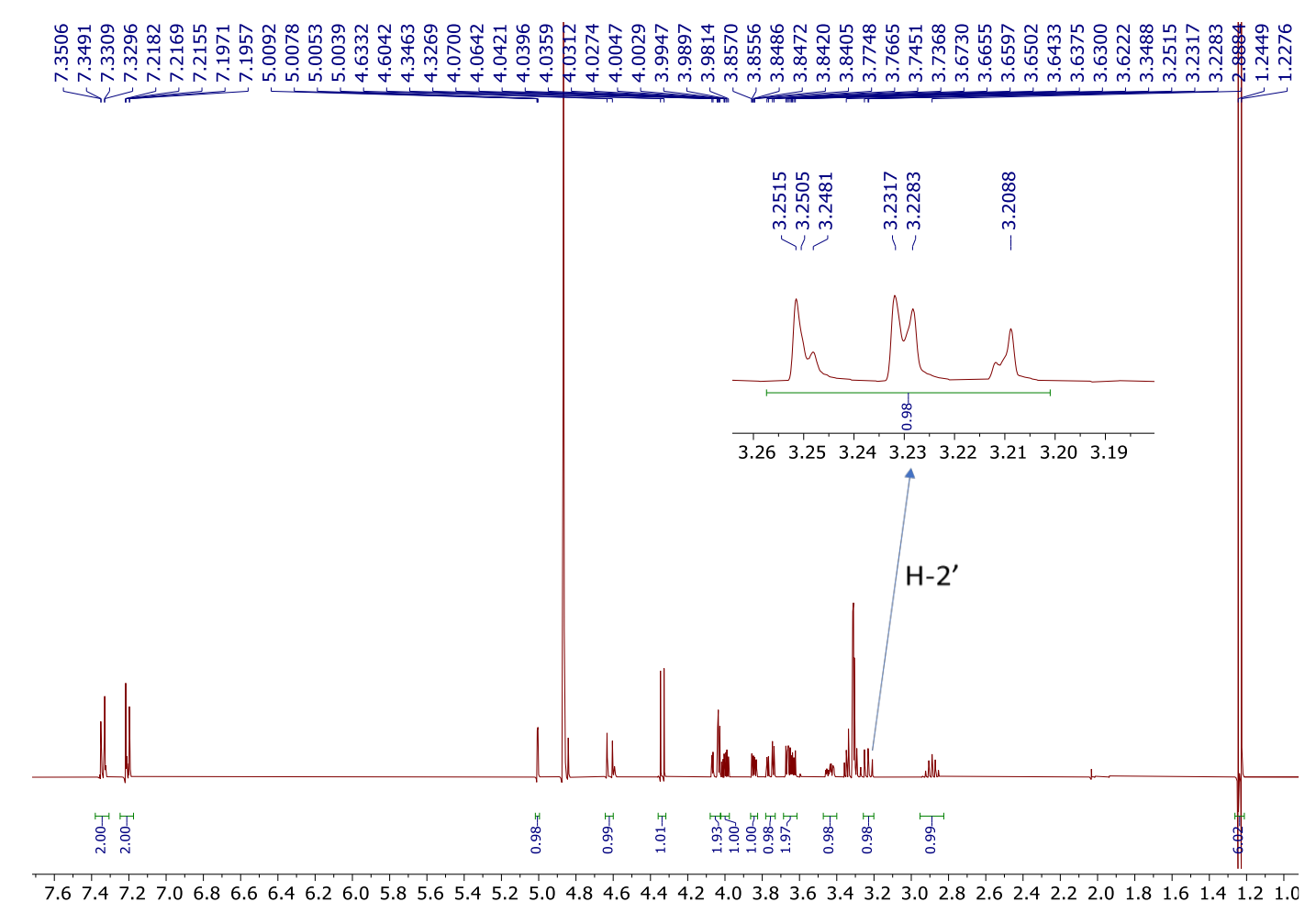

Figure S-21. ${ }^{1} \mathrm{H}$ NMR spectrum of 4 (400 MHz, $298 \mathrm{~K}$ ) in $\mathrm{CD}_{3} \mathrm{OD}$

\begin{tabular}{|c|c|c|c|c|c|c|c|}
\hline 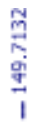 & 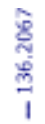 & 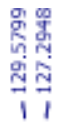 & 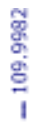 & 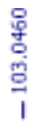 & 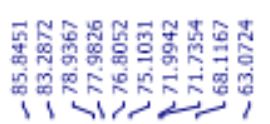 & $\begin{array}{l}8 \\
\text { o } \\
\text { g. } \\
\text { । }\end{array}$ & 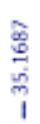 \\
\hline
\end{tabular}

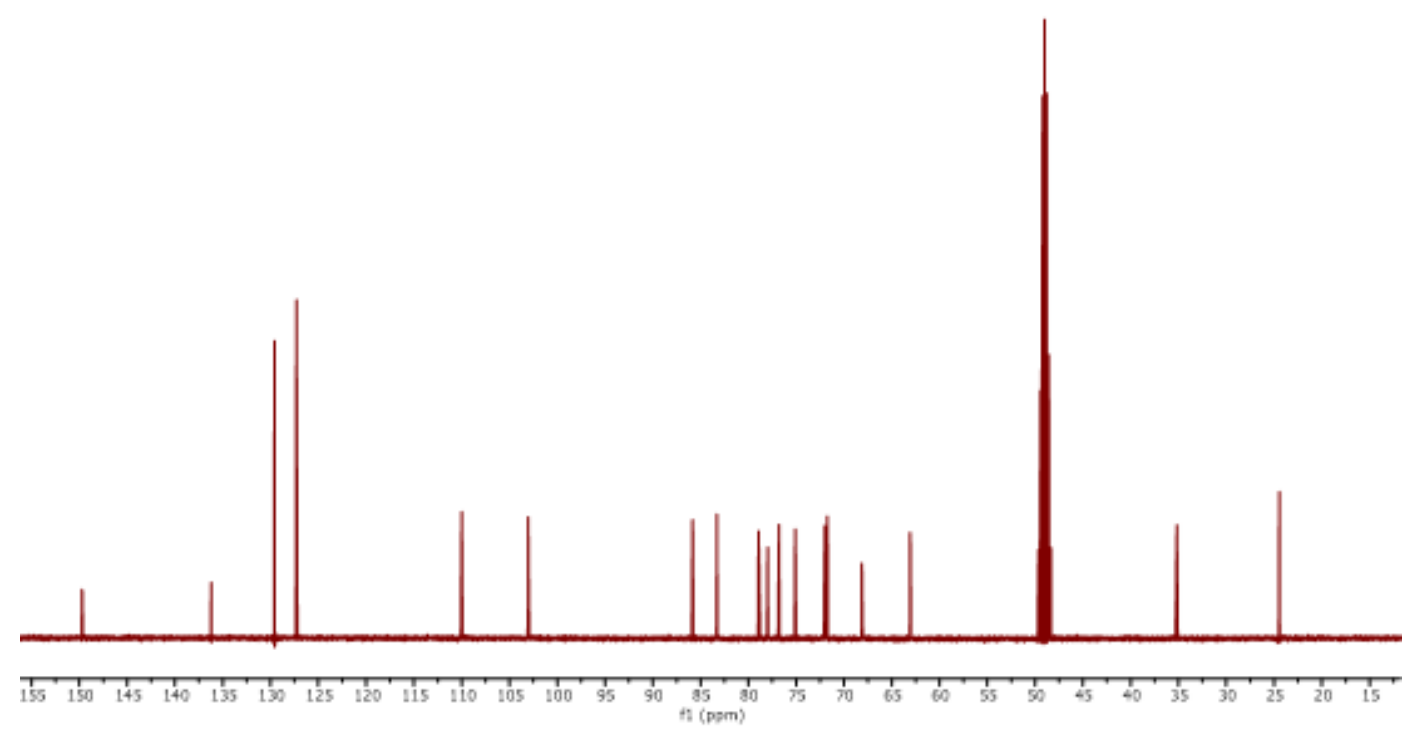

Figure S-22. ${ }^{13} \mathrm{C}$ NMR spectrum of $4(100 \mathrm{MHz}, 298 \mathrm{~K})$ in $\mathrm{CD}_{3} \mathrm{OD}$ 


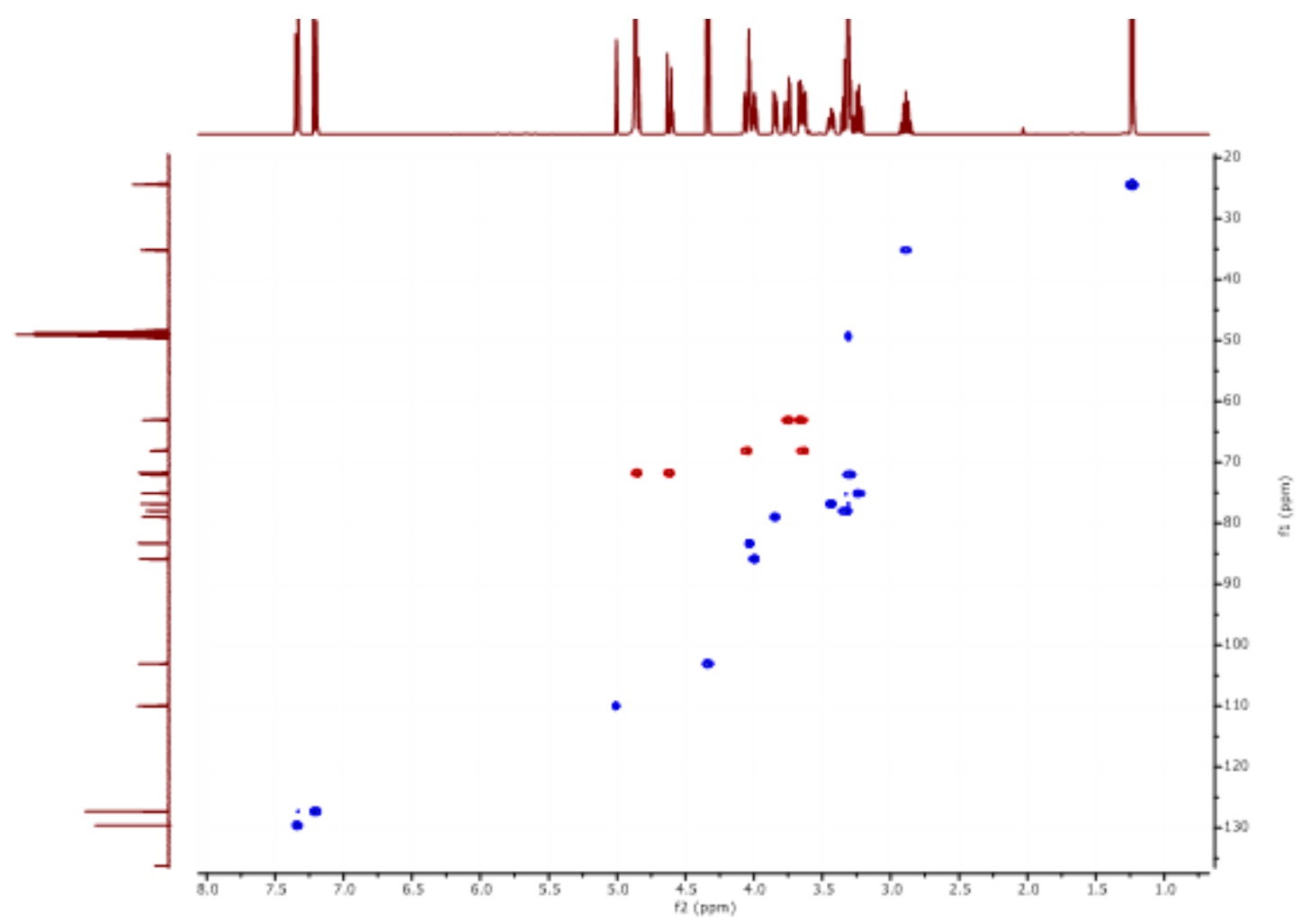

Figure S-23. HSQC spectrum of $4(400 \mathrm{MHz}, 298 \mathrm{~K})$ in $\mathrm{CD}_{3} \mathrm{OD}$

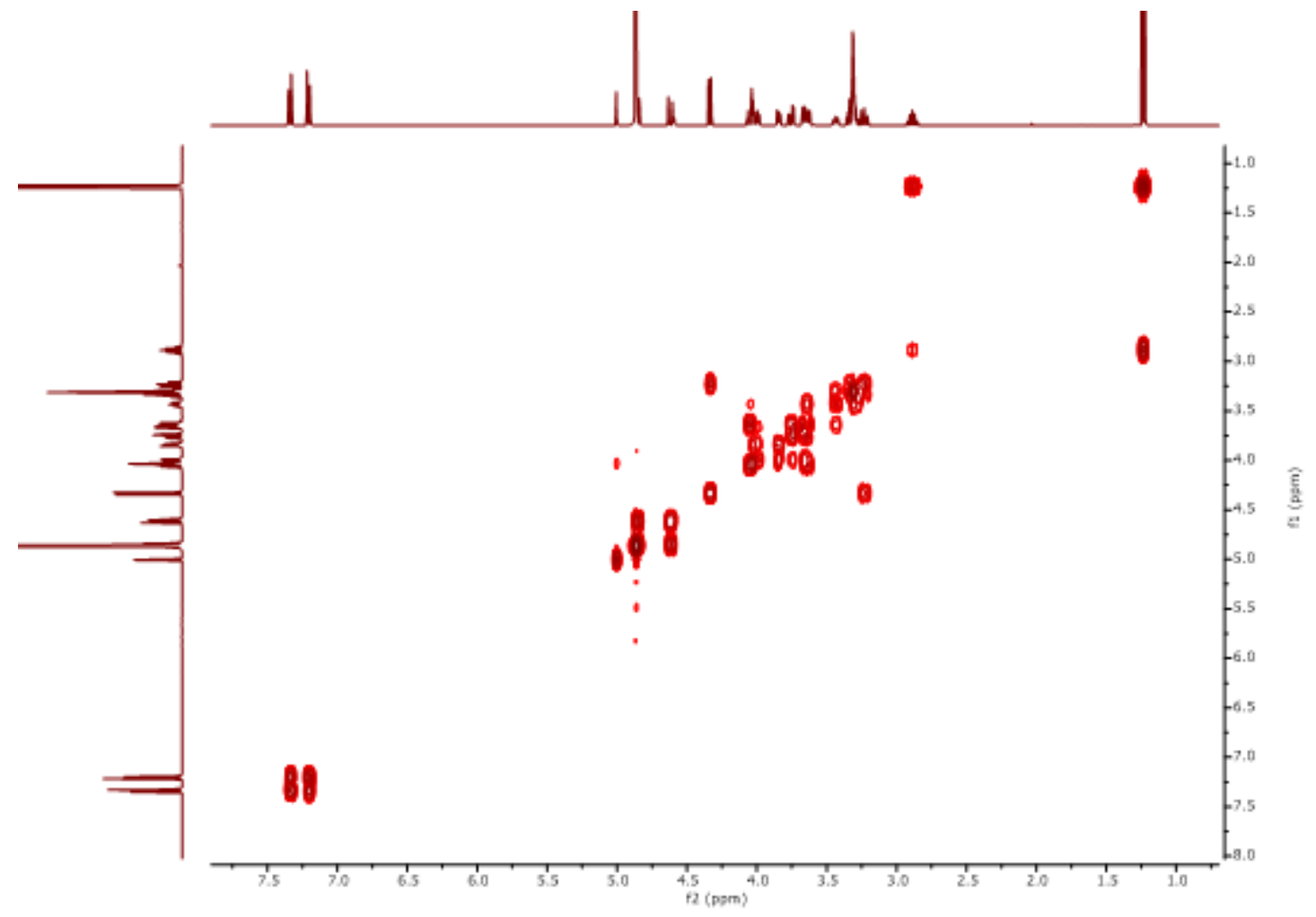

Figure S-24. COSY spectrum of 4 (400 MHz, $298 \mathrm{~K})$ in $\mathrm{CD}_{3} \mathrm{OD}$ 


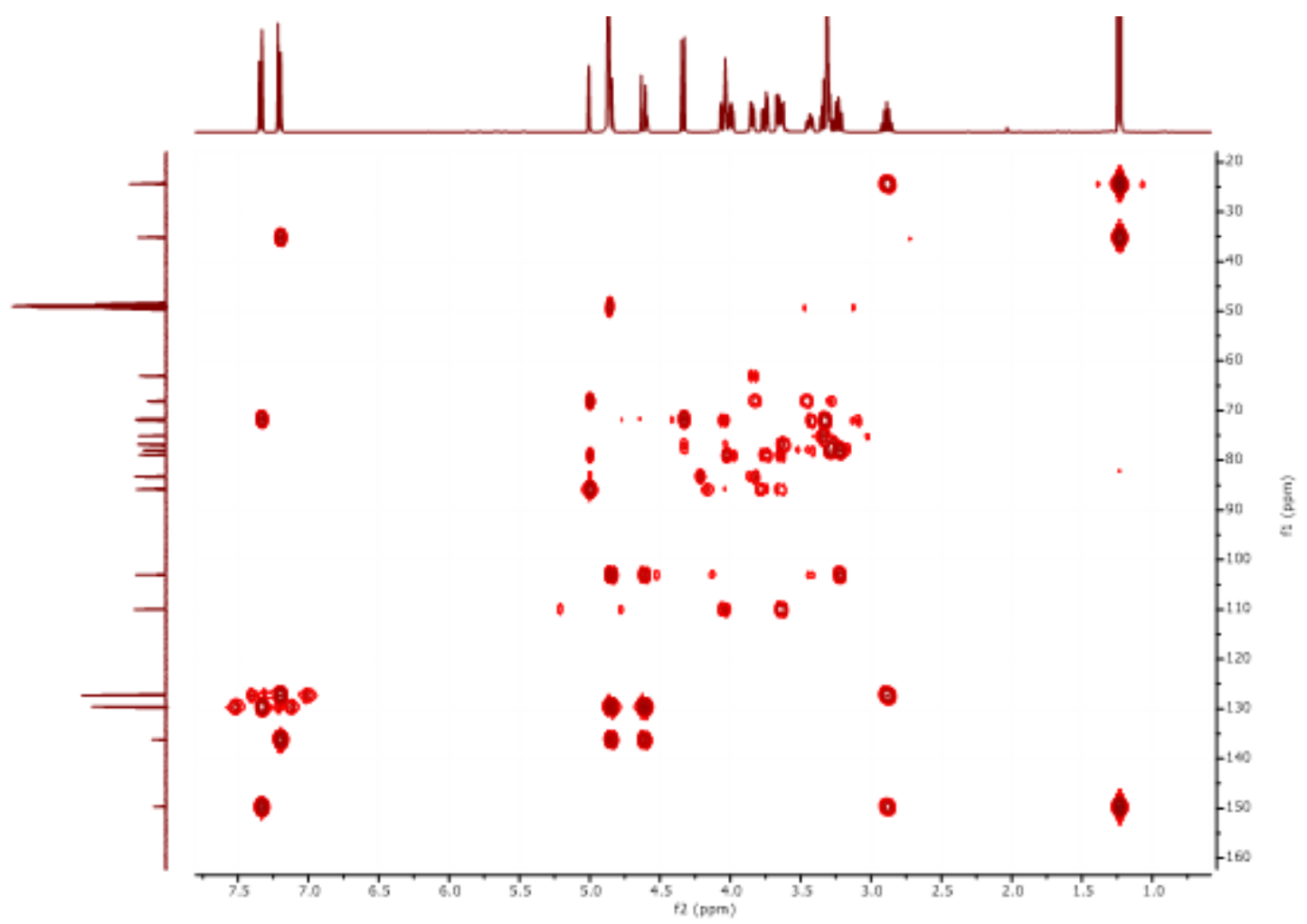

Figure S-25. HMBC spectrum of 4 (400 MHz, $298 \mathrm{~K}$ ) in $\mathrm{CD}_{3} \mathrm{OD}$ 


\section{Complete HiFSA Profile of Compound 4}

\footnotetext{
* NEW: the lines beginning by * are comment lines !

* To keep all the chemical shifts fixed during iteration

* replace "CHEMICAL SHIFTS(HZ):" by "..SHIFTS(HZ): fixed"

* The couplings can be fixed in the same way
}

CHEMICAL SHIFTS(PPM):

PROTON 2*SPIN $=1$ SPECIES $=1 \mathrm{H} \quad$ POPULATION $(\mathrm{Y})=0.07525$

H-1' / 1 4.336522 $1 * 1 * 1$ STAT=Y PRED= 4.336 RANGE= 0.378 WIDTH(Y)=0.668 RESP(Y)=1.0000 HSQC= C-1'

H-5' / $13.436161 \quad 1 * 1 * 1$ STAT=Y PRED= 3.436 RANGE= 0.283 WIDTH(Y)=1.093 RESP(Y)=1.0000 HSQC= C-5

H-4' / $13.2974981{ }^{*} 1^{*} 1$ STAT=Y PRED= 3.292 RANGE= 0.238 WIDTH(Y)=1.260 RESP(Y)=1.0000 HSQC $=\mathrm{C}^{\prime} \mathbf{4}^{\prime}$ H-3' / $13.3327891 * 1 * 1$ STAT=Y PRED= 3.337 RANGE= 0.213 WIDTH(Y)=0.660 RESP(Y)=1.0000 HSQC=C-3' H-2' / $13.2315491^{*} 1^{*} 1$ STAT=Y PRED= 3.229 RANGE=0.278 WIDTH $(\mathrm{Y})=0.840$ RESP(Y)=1.0000 HSQC $=\mathrm{C}^{\prime} \mathbf{2}^{\prime}$ H-6'a / 1 4.052752 1*1*1 STAT=Y PRED= 4.052 RANGE=0.378 WIDTH(Y)=1.145 RESP(Y)=1.0000 HSQC= C-6' $\mathrm{H}-6$ 'b $/ 13.6441181 * 1 * 1$ STAT $=Y$ PRED $=3.643$ RANGE $=0.293$ WIDTH $(\mathrm{Y})=1.153$ RESP $(\mathrm{Y})=1.0000 \mathrm{HSQC}=\mathrm{C}-\mathrm{6}^{\prime}$ H-3_5 / $17.2076781 * 2 * 1$ STAT=Y PRED= 7.206 RANGE=0.198 WIDTH(Y)=0.530 RESP(Y)=1.0000 HSQC=C-3_5 H-2_6 / $17.3393461 * 2 * 1$ STAT $=Y$ PRED $=7.339$ RANGE $=0.148$ WIDTH $(Y)=0.525$ RESP(Y)=1.0000 HSQC $=\mathrm{C}-2 \_6$ H-8 / $12.8882511 * 1 * 1$ STAT=Y PRED= 2.888 RANGE=0.188 WIDTH(Y)=1.235 RESP(Y)=1.0000 HSQC=C-8 H-9 / $11.2363941 * 1 * 3$ STAT=Y PRED= 1.236 RANGE $=0.148$ WIDTH $(\mathrm{Y})=0.419$ RESP $(\mathrm{Y})=1.0000 \mathrm{HSQC}=\mathrm{C}-9$ $\mathrm{H}-7 \mathrm{a} / 1$ 4.854059 $1 * 1 * 1$ STAT=Y PRED= 4.855 RANGE $=0.348$ WIDTH(Y)=0.684 RESP(Y)=1.0000 HSQC=C-7 $\mathrm{H}-7 \mathrm{~b} / 14.6197131 * 1 * 1$ STAT $=Y$ PRED $=4.618$ RANGE= $0.363 \mathrm{WIDTH}(\mathrm{Y})=0.854$ RESP(Y)=1.0000 HSQC= C-7 $\mathrm{H}-10 / 11.2361541 * 1 * 3$ STAT $=Y$ PRED $=1.236$ RANGE $=0.148$ WIDTH $(\mathrm{Y})=0.618$ RESP $(\mathrm{Y})=1.0000 \mathrm{HSQC}=\mathrm{C}-10$ H-4" / $13.9991581{ }^{*} 1 * 1$ STAT=Y PRED= 3.999 RANGE= 0.318 WIDTH(Y)=0.833 RESP(Y)=1.0000 HSQC=C-4" H-3" / $13.8450631 * 1 * 1$ STAT=Y PRED= 3.844 RANGE= 0.358 WIDTH(Y)=0.928 RESP(Y)=1.0000 HSQC= C-3" H-2" / 1 4.033431 1*1*1 STAT=Y PRED= 4.033 RANGE=0.328 WIDTH(Y)=0.745 RESP(Y)=1.0000 HSQC=C-2" $\mathrm{H}-1 " / 1 \quad 5.0065301 * 1 * 1$ STAT $=Y$ PRED $=5.006$ RANGE $=0.393$ WIDTH(Y)=0.908 RESP(Y)=1.0000 HSQC=C-1" H-5"a/ $13.7537861 * 1 * 1$ STAT=Y PRED= 3.755 RANGE= 0.208 WIDTH(Y)=1.107 RESP(Y)=1.0000 HSQC=C-5" H-5"b/ $13.6538701 * 1 * 1$ STAT=Y PRED= 3.651 RANGE= 0.198 WIDTH(Y)=1.153 RESP(Y)=1.0000 HSQC=C-5" MEOD_CH 2*SPIN $=1$ SPECIES $=1 \mathrm{H} \quad$ POPULATION $(Y)=0.24691$

MEOD / $23.3101001{ }^{*} 1 * 1$ STAT=Y PRED= 3.310 RANGE $=0.048$ WIDTH $(Y)=0.845 \operatorname{RESP}(\mathrm{Y})=1.0000$ MEOD_D $2 * S P I N=2$ SPECIES $=2 D$ POPULATION $(Y)=0.24691$

MEOD1 / $2-100.0000001 * 1 * 1$ STAT=Y PRED $=-99.999$ RANGE= 0.050 WIDTH(Y)=1.500 RESP(Y) $=1.0000$ MEOD2 / $2-100.0000001 * 1 * 1$ STAT=Y PRED $=-99.999$ RANGE $=0.050$ WIDTH $(Y)=1.500 \operatorname{RESP}(\mathrm{Y})=1.0000$ WATER_H $2 *$ SPIN $=1$ SPECIES $=1 \mathrm{H} \quad$ POPULATION $(\mathrm{Y})=0.67784$

WATER / $34.8672112 * 1 * 1$ STAT $=Y$ PRED $=4.867$ RANGE $=0.048$ WIDTH $(\mathrm{Y})=1.774 \operatorname{RESP}(\mathrm{Y})=1.0000$

COUPLING CONSTANTS(HZ):

J32_36 7.8539 J H-1' H-2' STAT=Y PRED= 7.766 RANGE $=2.000$

J33_34 9.5264 J H-5' H-4' STAT $=$ Y PRED $=9.797$ RANGE $=2.000$

J33_37 2.3784 J H-5' H-6'a STAT=Y PRED $=6.274$ RANGE $=2.040$

J33_38 6.1401 J H-5' H-6'b STAT $=Y$ PRED $=4.158$ RANGE= 2.980

J34_35 9.0696 J H-4' H-3' STAT=Y PRED= 8.959 RANGE= 2.000

J35_36 9.2788 J H-3' H-2' STAT=Y PRED= 9.288 RANGE $=2.000$

J37_38 -11.1813 J H-6'a H-6'b STAT $=Y$ PRED $=-11.330$ RANGE $=1.000$

J42_45 1.8539 J H-3_5 H-3_5 STAT=Y PRED $=1.948$ RANGE $=1.000$

J42_43 7.9037 J H-3_5 H-2_6 STAT $=Y$ PRED $=7.861$ RANGE $=1.000$

J42_44 $0.5067 \quad \mathrm{~J} \mathrm{H}-2 \_6 \mathrm{H}-3 \_5 \quad$ STAT $=Y$ PRED $=0.524$ RANGE $=1.000$

J45_46 -0.2136 J H-3_5 H-8 STAT=Y PRED $=-0.559$ RANGE= 1.000

J45_50 0.5554 J H-3_5 H-7a STAT $=Y$ PRED $=0.503$ RANGE $=1.000$

J45_51 0.1993 J H-3_5 H-7b STAT $=Y$ PRED $=0.434$ RANGE $=1.000$

J43_44 2.0114 J H-2_6 H-2_6 STAT $=Y$ PRED $=1.968$ RANGE $=1.000$

J44_46 0.2660 J H-2_6 H-8 STAT=Y PRED $=0.369$ RANGE $=1.000$

J44_50 -0.4732 J H-2_6 H-7a STAT $=Y$ PRED $=-0.703$ RANGE $=1.000$

J44_51 -0.5181 J H-2_6 H-7b STAT $=Y$ PRED $=-0.691$ RANGE $=1.000$

J46_47 6.9344 J H-8 $\mathrm{H}-9 \quad$ STAT=Y PRED= 6.944 RANGE= 1.000

J46_52 6.9247 J H-8 H-10 STAT=Y PRED $=6.944$ RANGE $=1.000$

J50_51 -11.2922 J H-7a H-7b STAT=Y PRED = -13.145 RANGE $=2.190$

J55_56 5.9784 J H-4" H-3" STAT=Y PRED= 3.470 RANGE= 2.000

J55_61 3.2964 J H-4" H-5"a STAT=Y PRED= 5.425 RANGE= 2.710

J55_62 5.3513 J H-4" H-5"b STAT=Y PRED= 3.283 RANGE= 2.000

J56_57 3.3617 J H-3" H-2" STAT=Y PRED= 6.299 RANGE $=2.810$

J56_58 0.5790 J H-3" H-1" STAT=Y PRED=0.581 RANGE= 2.810

J57_58 1.5251 J H-2" H-1" STAT=Y PRED= 5.007 RANGE= 2.440

J61_62 -11.8665 J H-5"a H-5"b STAT=Y PRED $=-12.098$ RANGE $=1.000$ 
JMEOD1 1.6442 J MEOD MEOD1 STAT $=Y$ PRED $=1.660$ RANGE $=0.050$

JMEOD2 1.6442 J MEOD MEOD2 STAT $=Y$ PRED $=1.660$ RANGE $=0.050$

CONTROL PARAMETERS:

Solvent $=$ none $($ def. $99 \%$ enriched)

$1.000=$ Concentration (vol\%, def $=1.0 \%)$

$0.00100000=$ Minimum line-intensity

$0.00100000=$ Diagonalization criterium (not in use)

$399.78220000=$ FIELD $(1 \mathrm{H}, \mathrm{MHz})$, used to transform shifts to ppms

$12.52428291=$ Left frequency $(\mathrm{ppm})$

$-2.47181680=$ Right frequency $(\mathrm{ppm})$

10.000 = Acquisition time (s, for QMTLS)

$0.000=$ Line-width (for modes D, P \& T, $0=$ use defaults)

$0.028587251=$ Data-point resolution $(\mathrm{Hz})$

$25.649=$ GAUSSIAN (\%, $0=$ use default from INF)

$-11.374=$ Dispersion contribution (\%, $0=$ use default from INF)

$0.00000000=$ Decoupling frequency (for DORES)

END of FILE

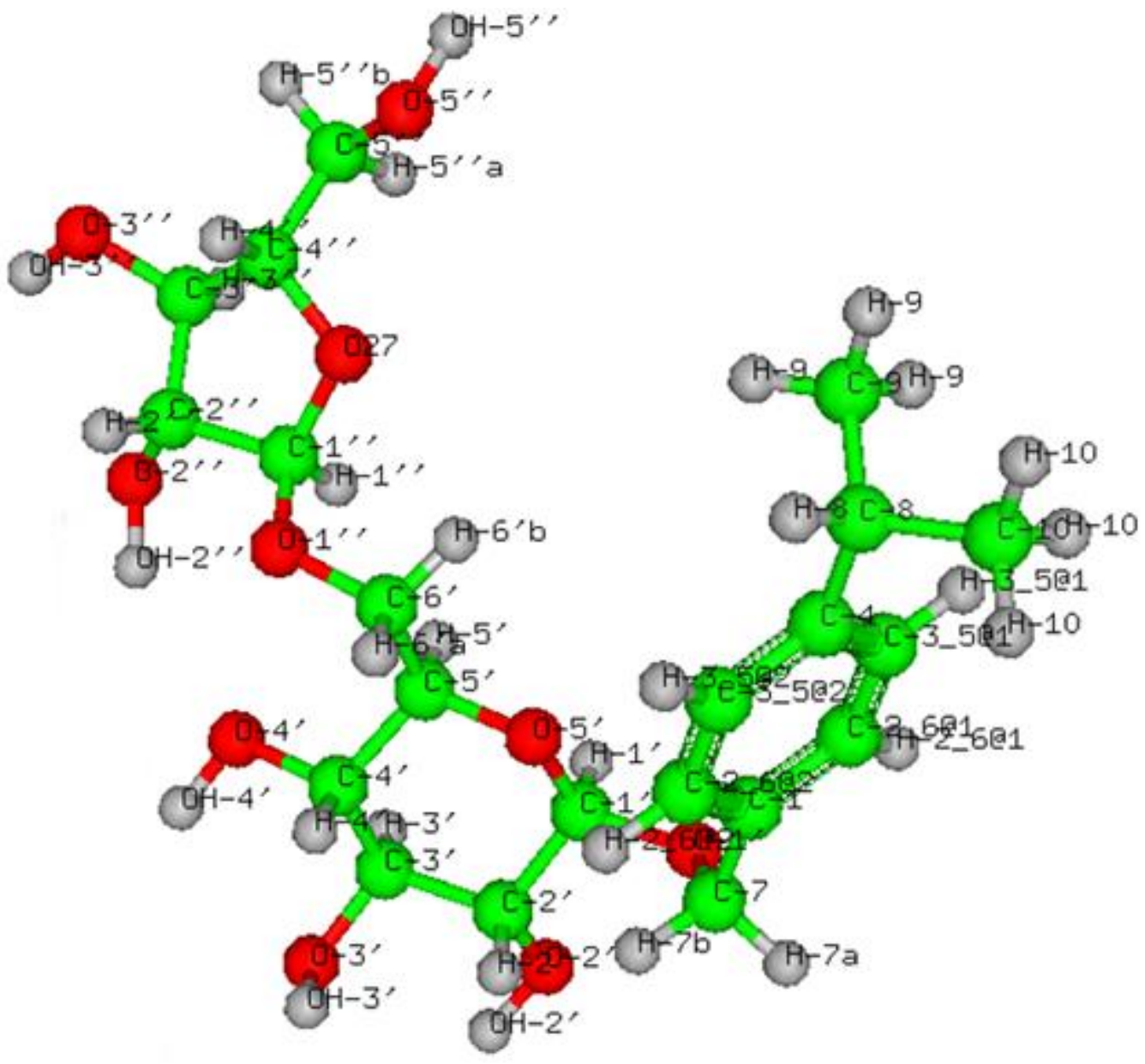




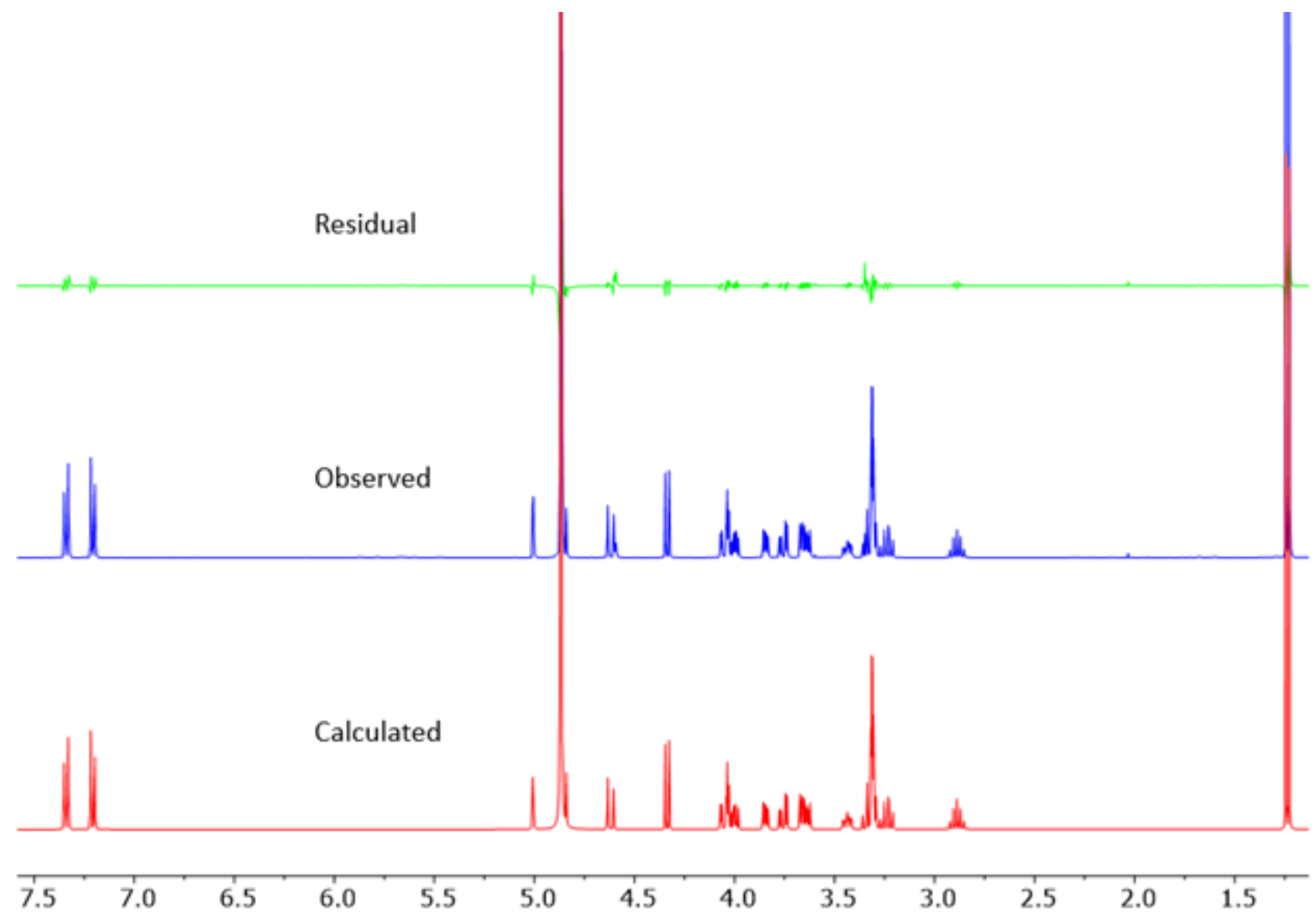

Figure S-26. The ${ }^{1} \mathrm{H}$ NMR fingerprint of 4 in $\mathrm{CD}_{3} \mathrm{OD}(400 \mathrm{MHz}, 298 \mathrm{~K})$ generated by PERCH 


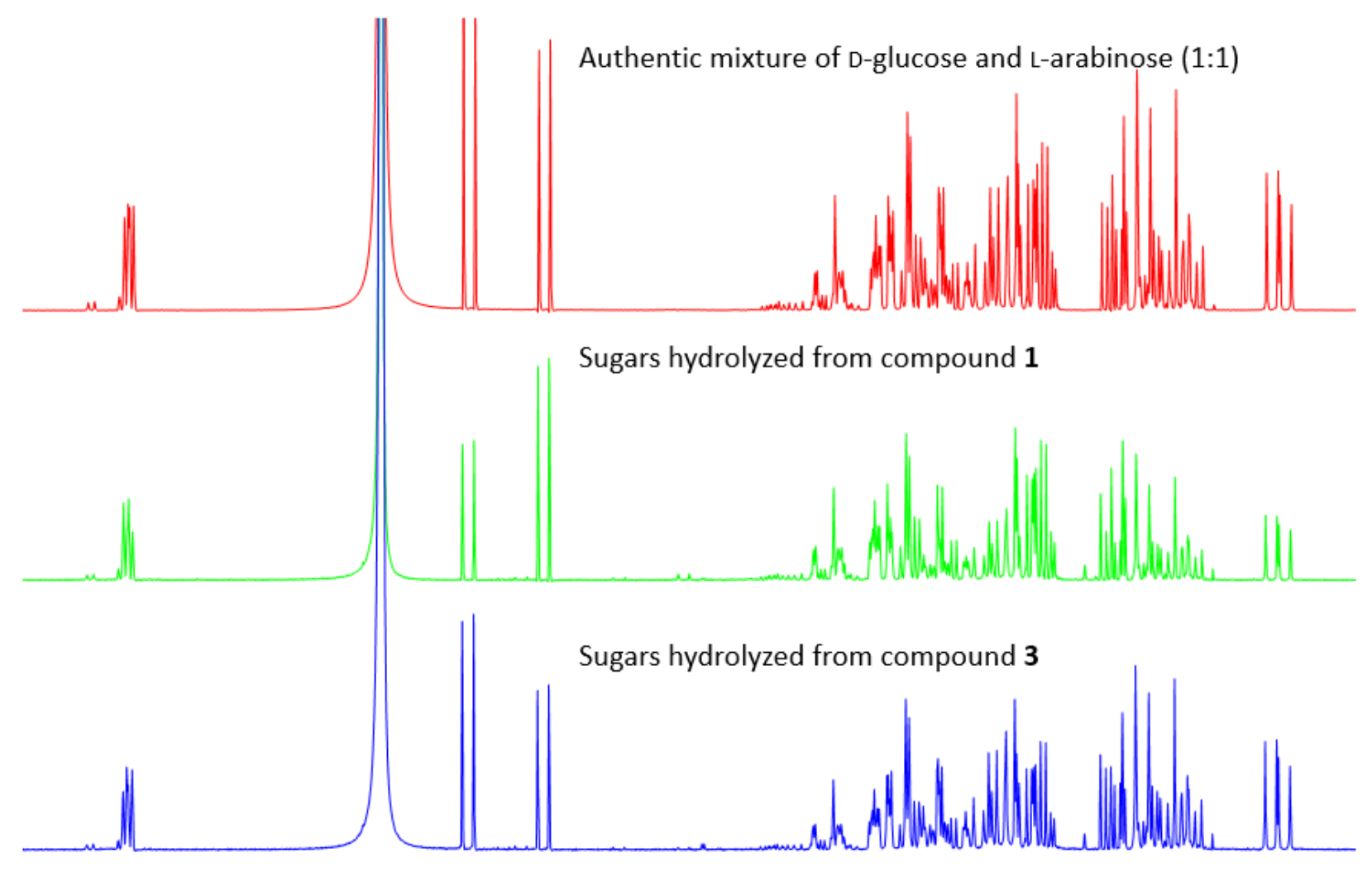

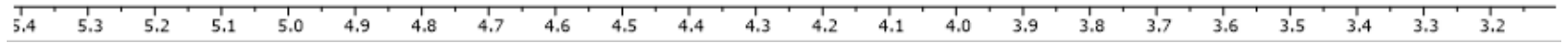

Figure S-27. ${ }^{1} \mathrm{H}$ NMR spectra comparison (400 MHz, $\left.298 \mathrm{~K}\right)$ between hydrolyzed sugars and authentic standard in $\mathrm{D}_{2} \mathrm{O}$

Table S-1. 100\% qHNMR Analysis of Compounds 1-4

\begin{tabular}{|c|c|}
\hline Compound & Purity (\%) \\
\hline $\mathbf{1}$ & 87.81 \\
\hline $\mathbf{2}$ & 98.46 \\
\hline 3 & 93.38 \\
\hline 4 & 99.20 \\
\hline
\end{tabular}


Table S-2. 100\% qHNMR Analysis of Detectable Impurities in Compounds 1-4

\begin{tabular}{|l|l|l|l|}
\hline \multicolumn{2}{|l|}{ compound } & detectable chemical shift $\left(\delta_{H}\right.$ in ppm) & content $(\% \mathrm{w} / \mathrm{w})$ \\
\hline \multirow{2}{*}{1} & impurity 1 & 0.87 & 6.85 \\
\cline { 2 - 5 } & impurity 2 & $1.74 ; 4.72$ & 3.32 \\
\cline { 2 - 5 } & impurity 3 & 1.95 & 2.09 \\
\hline \multirow{2}{*}{2} & impurity 1 & 0.87 & 1.30 \\
\cline { 2 - 5 } & impurity 2 & $1.74 ; 4.72$ & 0.24 \\
\hline \multirow{3}{*}{3} & impurity 1 & 5.00 & 3.77 \\
\cline { 2 - 5 } & impurity 2 & 0.94 & 1.56 \\
\cline { 2 - 5 } & impurity 3 & 0.68 & 0.44 \\
\hline & impurity 4 & 1.09 & 0.84 \\
\hline \multirow{2}{*}{$\mathbf{4}$} & impurity 1 & $1.59 ; 1.68$ & 0.02 \\
\hline & impurity 2 & 2.03 & 0.77 \\
\hline
\end{tabular}

Table S-3. 100\% qHNMR Analysis of Hydrolyzed Sugar Mixture in Compounds 1 and $\mathbf{3}$

\begin{tabular}{|c|l|l|}
\hline \multicolumn{2}{|c|}{ compound } & content (\% w/w) \\
\hline \multirow{2}{*}{$\mathbf{1}$} & D-glc & 39.84 \\
\cline { 2 - 3 } & L-ara & 57.82 \\
\hline \multirow{2}{*}{3} & D-glc & 58.30 \\
\cline { 2 - 3 } & L-ara & 40.24 \\
\hline
\end{tabular}

